\title{
EXISTENCE OF GLOBAL STRONG SOLUTION FOR THE COMPRESSIBLE NAVIER-STOKES SYSTEM AND THE KORTEWEG SYSTEM IN TWO-DIMENSION*
}

\author{
BORIS HASPOT ${ }^{\dagger}$
}

\begin{abstract}
This paper is dedicated to the study of viscous compressible barotropic fluids in dimension $N=2$. We address the question of the global existence of strong solutions with large initial data for compressible Navier-Stokes system and Korteweg system with friction. In the first case we are interested by slightly extending a famous result due to V. A. Vaigant and A. V. Kazhikhov in [34] concerning the existence of global strong solution in dimension two for a suitable choice of viscosity coefficient $\left(\mu(\rho)=\mu>0\right.$ and $\lambda(\rho)=\lambda \rho^{\beta}$ with $\left.\beta>3\right)$ in the torus. We are going to weaken the condition on $\beta$ by assuming only $\beta>2$ essentially by taking profit of commutator estimates introduced by Coifman et al in [7] and using a notion of effective velocity as in [34]. In the second case we study the existence of global strong solution with large initial data in the sense of the scaling of the equations for Korteweg system with degenerate viscosity coefficient and with friction term. It allows us in particular to prove the existence of global strong solution with large initial data in energy space when $N=2$. Let us point out that these results depend in an essential way on the structure of the viscosity coefficients.
\end{abstract}

Key words. Fluids mechanics, Navier Stokes equations, Korteweg system, harmonic analysis.

AMS subject classifications. 35A01, 35Q40, 35D35, 76N10.

1. Introduction. The motion of a general barotropic compressible fluid with capillary tensor is described by the following system, which can be derived from a Cahn-Hilliard free energy (see the pioneering work by J.- E. Dunn and J. Serrin in [9] and also in $[2,6,12])$ :

$$
\left\{\begin{array}{l}
\partial_{t} \rho+\operatorname{div}(\rho u)=0, \\
\partial_{t}(\rho u)+\operatorname{div}(\rho u \otimes u)-\operatorname{div}(2 \mu(\rho) D u)-\nabla(\lambda(\rho) \operatorname{div} u)+\nabla P(\rho)+a \rho u=\operatorname{div} K,
\end{array}\right.
$$

where $\operatorname{div} K$ is the capillary tensor which reads as follows:

$$
\operatorname{div} K=\nabla\left(\rho \kappa(\rho) \Delta \rho+\frac{1}{2}\left(\kappa(\rho)+\rho \kappa^{\prime}(\rho)\right)|\nabla \rho|^{2}\right)-\operatorname{div}(\kappa(\rho) \nabla \rho \otimes \nabla \rho) .
$$

The term $\operatorname{div} K$ allows to describe the variation of density at the interfaces between two phases, generally a mixture liquid-vapor. $P$ is a general increasing pressure term that we assume in the sequel under the form $P(\rho)=b \rho^{\gamma}$ with $b>0$ and $\gamma \geq 1$, $a \rho u$ is a friction term with $a>0$ (see [27]). $D(u)=\frac{1}{2}\left[\nabla u+{ }^{t} \nabla u\right]$ being the stress tensor, $\mu$ and $\lambda$ are the two Lamé viscosity coefficients depending on the density $\rho$ and satisfying:

$$
\mu>0 \text { and } 2 \mu+N \lambda \geq 0
$$

In the present paper, we are interested in dealing with two different situations:

- The case of the compressible Navier-Stokes system where we assume no capillarity et no friction, $\kappa(\rho)=0, a=0$ and where $\mu(\rho)=1$ is a constant and $\lambda(\rho)=\lambda \rho^{\beta}$ with $\beta \geq 2$.

\footnotetext{
*Received November 20, 2012; accepted for publication July 11, 2013.

†Ceremade UMR CNRS 7534, Université de Paris Dauphine, Place du Marchal DeLattre De Tassigny, 75775 Paris Cedex 16 (haspot@ceremade.dauphine.fr).
} 
- The case of Korteweg system with friction $a>0$ where the viscosity coefficients and the capillarity coefficient verify:

$$
\mu(\rho)=\mu \rho \quad \lambda(\rho)=0 \text { and } \kappa(\rho)=\frac{\kappa}{\rho} \text { with } \kappa>0, \mu>0 .
$$

In this paper we are interested in showing the importance of the viscosity coefficients when we want to deal with the existence of global strong solution for the system (1.6).

In the first case we would like to extend the famous result of global strong solution in two dimension in the torus discovered by V. A. Vaigant and A. V. Kazhikhov in [34]. Indeed in [34], the authors assume that $\lambda(\rho)=\lambda \rho^{\beta}$ with $\beta>3, \lambda>0$ and $\mu(\rho)=1$. Let us emphasize that a such choice on the viscosity coefficients allows to exhibits two different phenomena; the first one concerns the notion of effective velocity introduced by Lions in [26] which is crucial in this context for getting a priori estimates on the divergence and the rotational of the velocity; the second reason to choose such coefficient concerns the possibility to provide $L_{T}^{\infty}\left(L^{p}\left(\mathbb{T}^{2}\right)\right)$ estimates for any $p>1$ and any $T>0$ on the density. Indeed the viscosity coefficient $\lambda(\rho)$ take the role of a weight which contributes to furnish such estimates on the density (see the p1115 "Second a priori estimate for the density" in [34]). In the first part of this paper we wish to improve this result by assuming only $\lambda(\rho)=\lambda \rho^{\beta}$ with $\beta>2$ and $\lambda>0$. The key point will consist in using commutator estimates for dealing with term of the form $\left[R_{i j}, u_{j}\right]\left(\rho u_{i}\right)$ (we refer to [7] for such estimates, let us also mention that such commutator plays a crucial role in [26] for proving the global existence of weak solution for compressible Navier-Stokes equations).

In the second case we are interested in proving global existence of strong solution for the Korteweg system with friction term when the physical coefficients verify:

$$
\kappa(\rho)=\frac{\kappa}{\rho}, \kappa=\mu^{2}, \quad \gamma=1 \text { and } b=a \mu,
$$

with $\mu>0$. This system without friction has been widely studied this last year in particular concerning the existence of global weak solution and global strong solution with small initial data. We refer in particular to the following works $[5,8,16,17$, $18,19,20,22]$. Let us introduce a other notion of effective velocity used in particular in $[16,22]$ which allows us to simplify the system (1.6). Indeed by computation (see $[16])$, we obtain the simplified system:

$$
\left\{\begin{array}{l}
\partial_{t} \rho+\operatorname{div}(\rho v)-\mu \Delta \rho=0, \\
\rho \partial_{t} v+\rho u \cdot \nabla v-\operatorname{div}(\mu \rho \nabla \mathrm{v})+\mathrm{a} \rho \mathrm{v}=0,
\end{array}\right.
$$

with $v=u+\mu \nabla \ln \rho$ the effective velocity. For more details on the computation, we refer to [17]. When we write the system (1.4) in function of the momentum $m=\rho v$, the system reads as follows:

$$
\left\{\begin{array}{l}
\partial_{t} \rho+\operatorname{div} m-\mu \Delta \rho=0 \\
\partial_{t} m+\operatorname{div}\left(\frac{\mathrm{m}}{\rho} \otimes \mathrm{m}\right)-\mu \Delta \mathrm{m}+\mathrm{a} \mathrm{m}=0 .
\end{array}\right.
$$

In particular we observe that $(\rho,-\mu \nabla \ln \rho)$ when:

$$
\partial_{t} \rho-\mu \Delta \rho=0,
$$


is a particular global solution of the system (1.6).

REMARK 1. Let us mention that we can choose initial density which admits vacuum. In general it is not always possible to obtain global strong solution with initial density close from the vacuum.

We would like also to mention that as in the case of the paper of Vaigant and Kazhikhov [34], the viscosity coefficient plays a crucial role since they allows us to exhibit explicit irrotational solutions.

In the sequel we will be interested in working around this global particular solution (see remark 1) in order to prove the existence of global strong solution with large initial data on the irrotational part. More precisely we shall obtain global strong solution in critical space for the scaling of the equations with a small initial data $m_{0}$ and a large initial density $q_{0}=\rho_{0}-1$ (let us point out that it implies that via the definition of $v, u_{0}$ can be large). Let us briefly recall the notion of invariance by scaling of the equation and by what we mean by critical initial data. By critical, we mean that we want to solve the system (1.6) in functional spaces with invariant norm by the natural changes of scales which leave (1.6) invariant. More precisely in our case, the following transformation:

$$
(\rho(t, x), u(t, x)) \longrightarrow\left(\rho\left(l^{2} t, l x\right), l u\left(l^{2} t, l x\right)\right), \quad l \in \mathbb{R},
$$

verify this property, provided that the pressure term has been changed accordingly. In particular we can observe that $\dot{H}^{\frac{N}{2}} \times \dot{H}^{\frac{N}{2}-1}$ is a space invariant for the scaling of the equation, more generally such Besov spaces:

$$
\left(\rho_{0}-1\right) \in B_{p, 1}^{\frac{N}{p}}, u_{0} \in B_{p_{1}, 1}^{\frac{N}{p_{1}}-1},
$$

with $\left(p, p_{1}\right) \in[1,+\infty[$ are also available.

1.1. Results. Let us state the two main result of this paper. The first one is an improvement of the results of Vaigant and Kazhikhov [34].

THEOREM 1.1. Let us assume the following hypothesis on the viscosity coefficients:

$$
\mu(\rho)=1 \text { and } \lambda(\rho)=\lambda \rho^{\beta} \text { with } \beta>2 .
$$

Let $u_{0} \in H^{2}\left(\mathbb{T}^{2}\right), \rho_{0} \in W^{1, q}\left(\mathbb{T}^{2}\right)$ with $q>2$ and:

$$
0<c \leq \rho_{0}(x) \leq m<+\infty \quad \forall x \in \mathbb{T}^{2},
$$

then it exists a unique global strong solution to (1.6) such that:

$$
u \in W_{2}^{2,1}\left(Q_{T}\right) \text { and } \rho \in W_{q, \infty}^{1,1}\left(Q_{T}\right) \forall T>0,
$$

with $Q_{T}=(0, T) \times \mathbb{T}^{2}$.

REMARK 2. As mentioned above, the main point compared with the result of [34] concerns the fact that we can improve the range of $\beta$ by assuming only $\beta>2$. It would be possible also to improve the regularity condition on the initial data by working in Besov space invariant for the scaling of the system, but it is not the object of this paper. 
Let us mention that this result has also been improved independently by Huang and Li in [21] when $\beta>\frac{4}{3}$.

We are going to give our second result on Korteweg system with rcritical smallness condition on the initial data; before let us give the following definition:

Definition 1.1. We set $q=\rho-1, m=\rho v$.

In the following theorem we are dealing with the euclidian space $\mathbb{R}^{N}$ with $N \geq 2$.

TheOREM 1.2. Suppose that we are under the conditions (1.3). Assume that $m_{0} \in B_{2,1}^{\frac{N}{2}-1}$ and $q_{0} \in B_{2,1}^{\frac{N}{2}}$ with $\rho_{0} \geq c>0$. Then there exists a constant $\varepsilon_{0}$ depending on $\frac{1}{\rho_{0}}$ such that if:

$$
\left\|m_{0}\right\|_{B_{2,1}^{\frac{N}{2}-1}} \leq \varepsilon_{0}
$$

then there exists a unique global solution $(q, m)$ for system (1.5) with $\rho$ bounded away from zero and:

$$
q \in \widetilde{C}\left(\mathbb{R}^{+}, B_{2,1}^{\frac{N}{2}}\right) \cap L^{1}\left(\mathbb{R}^{+}, B_{2,1}^{\frac{N}{2}+2}\right) \quad \text { and } \quad m \in \widetilde{C}\left(\mathbb{R}^{+} ; B_{2,1}^{\frac{N}{2}-1}\right) \cap L^{1}\left(\mathbb{R}+, B_{2,1}^{\frac{N}{2}-1} \cap B_{2,1}^{\frac{N}{2}+1}\right) .
$$

REMARK 3. The main interest of this result consists in getting the existence of global strong solution with large initial density $q_{0} \in B_{2,1}^{\frac{N}{2}}$ and with large initial momentum $u_{0} \in B_{2,1}^{\frac{N}{2}-1}$. Indeed let us recall that $m=\rho v=\rho u+\mu \nabla \rho$ such that:

$$
u=\frac{m}{1+q}-\mu \nabla \ln (1+q) .
$$

We observe that $\nabla \ln \left(1+q_{0}\right)$ belongs in $B_{2,1}^{\frac{N}{2}-1}$ via the lemma 4 and can be choose arbitrary large whereas $\frac{m_{0}}{\rho_{0}}=v_{0}$ is small in $B_{2,1}^{\frac{N}{2}-1}$ via the lemma 4 and the smallness assumption on $m_{0}$. It implies that $u_{0}$ can be large in $B_{2,1}^{\frac{N}{2}-1}$ when $N \geq 2$.

Let us mention that when $N=2$ the initial data belongs in Besov space which are very close from the energy space.

REMARK 4. Let us mention that this result improves also the choice of the initial data compared with [8] where the initial density belongs in $B_{2,1}^{\frac{N}{2}-1} \cap B_{2,1}^{\frac{N}{2}}$. Indeed here via the introduction of the effective velocity we cancel out the coupling between density and velocity since the addition of the pressure and of the friction term gives a damping term in $v$. In other words at the difference with [8], the low frequencies on the density do not play any role here, that is why we assume only $q_{0} \in B_{2,1}^{\frac{N}{2}}$.

Let us give the plane of this paper, we shall remind in section 2 some auxiliary results of Gagliardo-Nirenberg's inequality and in section 3 the Litllewood-Paley theory. In section 4 and section 5 , we will prove different a priori estimates on the density and the velocity which show the theorem 1.1. We will conclude in section 6 by the proof of theorem 1.2.

Notation. In all the paper, $C$ will stand for a harmless constant, and we will sometimes use the notation $A \lesssim B$ equivalently to $A \leq C B$. 
2. Auxiliary assertions. We are going to recall some lemma which are also present in [34] and that we prefer to state for the sake of the completnes.

LEMMA 1. Let $\Omega \in \mathbb{R}^{N}$ be an arbitrary bounded domain satisfying the cone condition. Then the following inequality is valid for every function $u \in W^{1, m}(\Omega)$, $\int_{\Omega} u d x=0$

$$
\|u\|_{L^{q}(\Omega)} \leq C_{1}\|\nabla u\|_{L^{m}(\Omega)}^{\alpha}\|u\|_{L^{r}(\Omega)}^{1-\alpha}
$$

where $\alpha=\frac{\frac{1}{r}-\frac{1}{q}}{\frac{1}{r}-\frac{1}{m}+\frac{1}{n}}$, moreover if $m<n$ then $q \in\left[r, \frac{m n}{n-m}\right]$ for $r \leq \frac{m n}{n-m}$ and $q \in$ $\left[\frac{m n}{n-m}, r\right]$ for $r>\frac{m n}{n-m}$. If $m \geq n$ then $q \in[r,+\infty)$ is arbitrary; moreover if $m>n$ then equality (2.7) is also valid for $q=+\infty$.

Inequality (2.7) is a particular case of the more general inequalities proven in $[10,23,11]$. Let us mention that an inequality of the form $(2.7)$ is valid for the function of class $W^{1, m}(\Omega)$ when $M=\frac{1}{|\Omega|} \int_{\Omega} u d x$ is not null. It suffices to consider $v=u-M$ and apply inequality (2.7) to the function $v$. We obtain then the inequality

$$
\|u\|_{L^{q}(\Omega)} \leq C_{2}\left(\|\nabla u\|_{L^{m}(\Omega)}^{\alpha}\|u\|_{L^{r}(\Omega)}^{1-\alpha}+\|u\|_{L^{1}(\Omega)}\right),
$$

LEMMA 2. Let $\Omega \in \mathbb{R}^{2}$ be an arbitrary bounded domain satisfying the cone condition. Then every function $u \in W^{1, m}(\Omega)$ with $\int_{\Omega} u d x=0$ satisfies the inequality

$$
\|u\|_{L^{\frac{2 m}{2-m}}(\Omega)} \leq C_{3}(2-m)^{-\frac{1}{2}}\|\nabla u\|_{L^{m}(\Omega)}, \quad 1 \leq m<2
$$

where $C_{3}$ is a constant independent of $m$ and the function $u$.

For a proof of this inequality see $[35,32]$. The exact constant in inequality (2.9) is obtained in the article [32].

LEMmA 3. Let $\Omega \in \mathbb{R}^{2}$ be an arbitrary bounded domain satisfying the cone condition. Then for an arbitrary number $\varepsilon, 1 \geq 2 \varepsilon \geq 0$, every function $h \in W^{1, \frac{2 m}{m+\delta}}(\Omega)$, $m \geq 2,1 \geq \delta \geq 0$, satisfies the inequality

$$
\|h\|_{L^{2 m}(\Omega)} \leq C_{4}\left(\|h\|_{L^{1}(\Omega)}+m^{\frac{1}{2}}\|\nabla h\|_{L^{\frac{2 m}{m+\delta}}(\Omega)}^{1-s}\|h\|_{L^{2(1-\varepsilon)}(\Omega)}^{s}\right)
$$

where $s=(1-\varepsilon) \frac{1-\delta}{m-\delta(1-\varepsilon)}$ and $C_{4}$ is a positive constant independent of $m, \varepsilon, \delta$ and the function $h$.

3. Littlewood-Paley theory and Besov spaces. Throughout the paper, $C$ stands for a constant whose exact meaning depends on the context. The notation $A \lesssim B$ means that $A \leq C B$. For all Banach space $X$, we denote by $C([0, T], X)$ the set of continuous functions on $[0, T]$ with values in $X$. For $p \in[1,+\infty]$, the notation $L^{p}(0, T, X)$ or $L_{T}^{p}(X)$ stands for the set of measurable functions on $(0, T)$ with values in $X$ such that $t \rightarrow\|f(t)\|_{X}$ belongs to $L^{p}(0, T)$. Littlewood-Paley decomposition corresponds to a dyadic decomposition of the space in Fourier variables. We can use for instance any $\varphi \in C^{\infty}\left(\mathbb{R}^{N}\right)$, supported in $\mathcal{C}=\left\{\xi \in \mathbb{R}^{N} / \frac{3}{4} \leq|\xi| \leq \frac{8}{3}\right\}$ such that:

$$
\sum_{l \in \mathbb{Z}} \varphi\left(2^{-l} \xi\right)=1 \text { if } \xi \neq 0
$$


Denoting $h=\mathcal{F}^{-1} \varphi$, we then define the dyadic blocks by:

$$
\Delta_{l} u=\varphi\left(2^{-l} D\right) u=2^{l N} \int_{\mathbb{R}^{N}} h\left(2^{l} y\right) u(x-y) d y \text { and } S_{l} u=\sum_{k \leq l-1} \Delta_{k} u .
$$

Formally, one can write that:

$$
u=\sum_{k \in \mathbb{Z}} \Delta_{k} u
$$

This decomposition is called homogeneous Littlewood-Paley decomposition.

\subsection{Homogeneous Besov spaces and first properties.}

Definition 3.2. For $s \in \mathbb{R}, p \in[1,+\infty], q \in[1,+\infty]$, and $u \in \mathcal{S}^{\prime}\left(\mathbb{R}^{N}\right)$ we set:

$$
\|u\|_{B_{p, q}^{s}}=\left(\sum_{l \in \mathbb{Z}}\left(2^{l s}\left\|\Delta_{l} u\right\|_{L^{p}}\right)^{q}\right)^{\frac{1}{q}} .
$$

The Besov space $B_{p, q}^{s}$ is the set of temperate distribution u such that $\|u\|_{B_{p, q}^{s}}<+\infty$.

Proposition 3.1. The following properties holds:

1. there exists a constant universal $C$ such that:

$C^{-1}\|u\|_{B_{p, r}^{s}} \leq\|\nabla u\|_{B_{p, r}^{s-1}} \leq C\|u\|_{B_{p, r}^{s}}$.

2. If $p_{1}<p_{2}$ and $r_{1} \leq r_{2}$ then $B_{p_{1}, r_{1}}^{s} \hookrightarrow B_{p_{2}, r_{2}}^{s-N\left(1 / p_{1}-1 / p_{2}\right)}$.

Let now recall a few product laws in Besov spaces coming directly from the paradifferential calculus of J-M. Bony (see [4]) and rewrite in [3].

Proposition 3.2. We have the following laws of product:

- For all $s \in \mathbb{R},(p, r) \in[1,+\infty]^{2}$ we have:

$$
\|u v\|_{B_{p, r}^{s}} \leq C\left(\|u\|_{L^{\infty}}\|v\|_{B_{p, r}^{s}}+\|v\|_{L^{\infty}}\|u\|_{B_{p, r}^{s}}\right) .
$$

- Let $\left(p, p_{1}, p_{2}, r, \lambda_{1}, \lambda_{2}\right) \in[1,+\infty]^{2}$ such that: $\frac{1}{p} \leq \frac{1}{p_{1}}+\frac{1}{p_{2}}, p_{1} \leq \lambda_{2}, p_{2} \leq \lambda_{1}$, $\frac{1}{p} \leq \frac{1}{p_{1}}+\frac{1}{\lambda_{1}}$ and $\frac{1}{p} \leq \frac{1}{p_{2}}+\frac{1}{\lambda_{2}}$. We have then the following inequalities: if $s_{1}+s_{2}+N \inf \left(0,1-\frac{1}{p_{1}}-\frac{1}{p_{2}}\right)>0, s_{1}+\frac{N}{\lambda_{2}}<\frac{N}{p_{1}}$ and $s_{2}+\frac{N}{\lambda_{1}}<\frac{N}{p_{2}}$ then:

$$
\|u v\|_{B_{p, r}^{s_{1}+s_{2}-N\left(\frac{1}{p_{1}}+\frac{1}{p_{2}}-\frac{1}{p}\right)}} \lesssim\|u\|_{B_{p_{1}, r}^{s_{1}}}\|v\|_{B_{p_{2}, \infty}^{s_{2}}}
$$

when $s_{1}+\frac{N}{\lambda_{2}}=\frac{N}{p_{1}}$ (resp $\left.s_{2}+\frac{N}{\lambda_{1}}=\frac{N}{p_{2}}\right)$ we replace $\|u\|_{B_{p_{1}, r}^{s_{1}}}\|v\|_{B_{p_{2}, \infty}^{s_{2}}}$ (resp $\|v\|_{B_{p_{2}, \infty}^{s_{2}}}$ ) by $\|u\|_{B_{p_{1}, 1}^{s_{1}}}\|v\|_{B_{p_{2}, r}^{s_{2}, r}}\left(\right.$ resp $\left.\|v\|_{B_{p_{2}, \infty}^{s_{2}} \cap L^{\infty}}\right)$, if $s_{1}+\frac{N}{\lambda_{2}}=\frac{N}{p_{1}}$ and $s_{2}+$ $\frac{N}{\lambda_{1}}=\frac{N}{p_{2}}$ we take $r=1$.

If $s_{1}+s_{2}=0, s_{1} \in\left(\frac{N}{\lambda_{1}}-\frac{N}{p_{2}}, \frac{N}{p_{1}}-\frac{N}{\lambda_{2}}\right]$ and $\frac{1}{p_{1}}+\frac{1}{p_{2}} \leq 1$ then:

$$
\|u v\|_{B_{p, \infty}^{-N\left(\frac{1}{p_{1}}+\frac{1}{p_{2}}-\frac{1}{p}\right)}} \lesssim\|u\|_{B_{p_{1}, 1}^{s_{1}}}\|v\|_{B_{p_{2}, \infty}^{s_{2}}} .
$$

If $|s|<\frac{N}{p}$ for $p \geq 2$ and $-\frac{N}{p^{\prime}}<s<\frac{N}{p}$ else, we have:

$$
\|u v\|_{B_{p, r}^{s}} \leq C\|u\|_{B_{p, r}^{s}}\|v\|_{B_{p, \infty}^{\frac{N}{p}} \cap L^{\infty}} .
$$


The study of non stationary PDE's requires space of type $L^{\rho}(0, T, X)$ for appropriate Banach spaces $X$. In our case, we expect $X$ to be a Besov space, so that it is natural to localize the equation through Littlewood-Payley decomposition. But, in doing so, we obtain bounds in spaces which are not type $L^{\rho}(0, T, X)$ (except if $\left.r=p\right)$. We are now going to define the spaces of CheminLerner in which we will work, which are a refinement of the spaces $L_{T}^{\rho}\left(B_{p, r}^{s}\right)$.

Definition 3.3. Let $\rho \in[1,+\infty], T \in[1,+\infty]$ and $s_{1} \in \mathbb{R}$. We set:

$$
\|u\|_{\widetilde{L}_{T}^{\rho}\left(B_{p, r}^{\left.s_{1}\right)}\right.}=\left(\sum_{l \in \mathbb{Z}} 2^{l r s_{1}}\left\|\Delta_{l} u(t)\right\|_{L^{\rho}\left(L^{p}\right)}^{r}\right)^{\frac{1}{r}} .
$$

We then define the space $\widetilde{L}_{T}^{\rho}\left(B_{p, r}^{s_{1}}\right)$ as the set of temperate distribution u over $(0, T) \times$ $\mathbb{R}^{N}$ such that $\|u\|_{\widetilde{L}_{T}^{\rho}\left(B_{p, r}^{\left.s_{1}\right)}\right.}<+\infty$.

We set $\widetilde{C}_{T}\left(\widetilde{B}_{p, r}^{s_{1}}\right)=\widetilde{L}_{T}^{\infty}\left(\widetilde{B}_{p, r}^{s_{1}}\right) \cap \mathcal{C}\left([0, T], B_{p, r}^{s_{1}}\right)$. Let us emphasize that, according to Minkowski inequality, we have:

$$
\|u\|_{\widetilde{L}_{T}^{\rho}\left(B_{p, r}^{s_{1}}\right)} \leq\|u\|_{L_{T}^{\rho}\left(B_{p, r}^{s_{1}}\right)} \text { if } r \geq \rho, \quad\|u\|_{\widetilde{L}_{T}^{\rho}\left(B_{p, r}^{s_{1}}\right)} \geq\|u\|_{L_{T}^{\rho}\left(B_{p, r}^{s_{1}}\right)} \text { if } r \leq \rho .
$$

REMARK 5. It is easy to generalize proposition 3.2, to $\widetilde{L}_{T}^{\rho}\left(B_{p, r}^{s_{1}}\right)$ spaces. The indices $s_{1}, p, r$ behave just as in the stationary case whereas the time exponent $\rho$ behaves according to Hölder inequality.

In the sequel we will need of composition lemma in $\widetilde{L}_{T}^{\rho}\left(B_{p, r}^{s}\right)$ spaces.

LEMMA 4. Let $s>0,(p, r) \in[1,+\infty]$ and $u \in \widetilde{L}_{T}^{\rho}\left(B_{p, r}^{s}\right) \cap L_{T}^{\infty}\left(L^{\infty}\right)$.

1. Let $F \in W_{l o c}^{[s]+2, \infty}\left(\mathbb{R}^{N}\right)$ such that $F(0)=0$. Then $F(u) \in \widetilde{L}_{T}^{\rho}\left(B_{p, r}^{s}\right)$. More precisely there exists a function $C$ depending only on $s, p, r, N$ and $F$ such that:

$$
\|F(u)\|_{\widetilde{L}_{T}^{\rho}\left(B_{p, r}^{s}\right)} \leq C\left(\|u\|_{L_{T}^{\infty}\left(L^{\infty}\right)}\right)\|u\|_{\widetilde{L}_{T}^{\rho}\left(B_{p, r}^{s}\right)}
$$

2. If $v, u \in \widetilde{L}_{T}^{\rho}\left(B_{p, r}^{s}\right) \cap L_{T}^{\infty}\left(L^{\infty}\right)$ and $G \in W_{\text {loc }}^{[s]+3, \infty}\left(\mathbb{R}^{N}\right)$ then $G(u)-G(v)$ belongs to $\widetilde{L}_{T}^{\rho}\left(B_{p}^{s}\right)$ and there exists a constant $C$ depending only of $s, p, N$ and $G$ such that:

$$
\begin{aligned}
& \|G(u)-G(v)\|_{\widetilde{L}_{T}^{\rho}\left(B_{p, r}^{s}\right)} \\
\leq & C\left(\|u\|_{L_{T}^{\infty}\left(L^{\infty}\right)},\|v\|_{L_{T}^{\infty}\left(L^{\infty}\right)}\right)\left(\| v - u \| _ { \widetilde { L } _ { T } ^ { \rho } ( B _ { p , r } ^ { s } ) } \left(1+\|u\|_{L_{T}^{\infty}\left(L^{\infty}\right)}\right.\right. \\
& \left.\left.+\|v\|_{L_{T}^{\infty}\left(L^{\infty}\right)}\right)+\|v-u\|_{L_{T}^{\infty}\left(L^{\infty}\right)}\left(\|u\|_{\widetilde{L}_{T}^{\rho}\left(B_{p, r}^{s}\right)}+\|v\|_{\widetilde{L}_{T}^{\rho}\left(B_{p, r}^{s}\right)}\right)\right) .
\end{aligned}
$$

Let us now give some estimates for the heat equation:

Proposition 3.3. Let $s \in \mathbb{R},(p, r) \in[1,+\infty]^{2}$ and $1 \leq \rho_{2} \leq \rho_{1} \leq+\infty$. Assume that $u_{0} \in B_{p, r}^{s}$ and $f \in \widetilde{L}_{T}^{\rho_{2}}\left(B_{p, r}^{s-2+2 / \rho_{2}}\right)$. Let $u$ be a solution of:

$$
\left\{\begin{array}{l}
\partial_{t} u-\mu \Delta u=f \\
u_{t=0}=u_{0}
\end{array}\right.
$$


Then there exists $C>0$ depending only on $N, \mu, \rho_{1}$ and $\rho_{2}$ such that:

$$
\|u\|_{\widetilde{L}_{T}^{\rho_{1}}\left(\widetilde{B}_{p, r}^{s+2 / \rho_{1}}\right)} \leq C\left(\left\|u_{0}\right\|_{B_{p, r}^{s}}+\mu^{\frac{1}{\rho_{2}}-1}\|f\|_{\widetilde{L}_{T}^{\rho_{2}\left(B_{p, r}^{s-2+2 / \rho_{2}}\right)}}\right) .
$$

If in addition $r$ is finite then $u$ belongs to $C\left([0, T], B_{p, r}^{s}\right)$.

4. Proof of Theorem 1.1. In the sequel we shall work on the torus $\Omega=\mathbb{T}^{2}$. Let us start with recalling the energy estimate, when we multiply the momentum equation we get:

$$
\begin{aligned}
& \int_{\Omega}\left(\rho|u|^{2}(t, x)+\pi(\rho)(t, x)\right) d x+\int_{0}^{t} \int_{\Omega}|\nabla u|^{2}(s, x) d s d x \\
& +\int_{0}^{t} \int_{\Omega}(1+\lambda(\rho)(s, x))(\operatorname{div} u)^{2}(s, x) d s d x \leq \int_{\Omega}\left(\rho_{0}(x)\left|u_{0}(x)\right|^{2}+\Pi\left(\rho_{0}\right)(x)\right) d x
\end{aligned}
$$

with $\pi$ defined as follows:

$$
\pi(\rho)=a\left(\frac{1}{\gamma-1}\left(\rho^{\gamma}-\rho\right)-\rho+1\right) \text { for } \gamma>1 .
$$

Let us recall that $P^{\prime}(\rho)=\rho \pi^{\prime \prime}(\rho)$ what implies by convexity that $\pi(\rho) \geq 0$. Finally as we assume that:

$$
C_{1}=\int_{\Omega}\left(\frac{1}{2} \rho_{0}(x)\left|u_{0}(x)\right|^{2}+\pi\left(\rho_{0}\right)(x)+\rho_{0}(x)\right) d x
$$

is finite, we obtain at least formally (if $\rho$ and $u$ are enough regular for performing integration by parts) by energy estimate (4.15) and via the transport equation that:

$$
\begin{aligned}
\int_{\Omega}\left(\rho|u|^{2}(t, x)+\pi(\rho)(t, x)\right. & +\rho(t, x)) d x+\int_{0}^{t} \int_{\Omega}|\nabla u|^{2}(s, x) d s d x \\
& +\int_{0}^{t} \int_{\Omega}(1+\lambda(\rho)(s, x))(\operatorname{div} u)^{2}(s, x) d s d x \leq C_{1}
\end{aligned}
$$

Let us now explain how to get $L^{2}((0, T) \times \Omega)$ estimates on $u$, we are going to follow Lions in [26] p4. Indeed by the momentum equation we have:

$$
\left|\int_{\Omega} \rho u(t, x) d x\right|=\left|\int_{\Omega} \rho_{0} u_{0}(x) d x\right| \leq\left\|\rho_{0} u_{0}\right\|_{L^{1}(\Omega)} .
$$

Next we use the Poincaré-Wirtinger inequality and we have:

Hence for all $t \geq 0$ :

$$
\left|\int_{\Omega} \rho(t, x)\left[u(t, x)-\int_{\Omega} u(t, y) d y\right] d x\right| \leq C\|\rho(t, \cdot)\|_{L^{\gamma}}\|\nabla u\|_{L^{2}(\Omega)} .
$$

$$
\left|\int_{\Omega} u(t, x) d x\right| \leq \frac{1}{\left(\int_{\Omega} \rho_{0} d x\right)}\left(\left\|\rho_{0} u_{0}\right\|_{L^{1}(\Omega)}+C\|\rho(t, \cdot)\|_{L^{\gamma}}\|\nabla u\|_{L^{2}(\Omega)}\right) .
$$

We conclude by Poincaré-Wirtinger inequality which implies that $\left|\int_{\Omega} u(t, x) d x\right|+$ $\|\nabla u\|_{L^{2}(\Omega)}$ is an equivalent norm to the usual one in $H^{1}(\Omega)$.

Now we are just going to explain where in the proof we can slightly improve the range of the coefficient $\beta$ in [34]. One of the main point of the proof in [34] consists in getting a priori estimates on the density in $L^{\infty}\left(L^{p}\left(\mathbb{T}^{2}\right)\right)$ for any $p>1$. This is possible due to the viscosity coefficient $\lambda(\rho)=\rho^{\beta}$ which provide such estimate at least if $\beta$ is large enough. Let us follow the arguments of the proof of [34] and explain where by commutators estimates we can weaken the hypothesis $\beta>3$. 
5. A priori estimates on the density and the velocity. First as in [34], we are going to recall some estimates for solutions to the following two Neumann problems:

$$
\begin{gathered}
\Delta \xi=\operatorname{div}(\rho u), \quad \int_{\Omega} \xi d x=0,\left.\quad \partial_{x_{1}} \xi\right|_{x_{1}=0, x_{1}=1}=\left.\partial_{x_{2}} \xi\right|_{x_{2}=0, x_{2}=1}=0 . \\
\Delta \eta=\operatorname{div}(\operatorname{div}(\rho u \otimes u)), \quad \int_{\Omega} \eta d x=0,\left.\quad \partial_{x_{1}} \eta\right|_{x_{1}=0, x_{1}=1}=\left.\partial_{x_{2}} \eta\right|_{x_{2}=0, x_{2}=1}=0 .
\end{gathered}
$$

Therefore by [25] we have solution to the problems (5.17) and (5.18), whereas the estimates for singular integrals in [30] provide the following inequalities:

$$
\begin{aligned}
& \left\|\nabla(\Delta)^{-1} \operatorname{div}(\rho u)\right\|_{L^{2 m}} \lesssim m\|\rho u\|_{L^{2 m}}, \quad 1 \leq m<+\infty \\
& \left\|\nabla(\Delta)^{-1} \operatorname{div}(\rho u)\right\|_{L^{2-r}} \lesssim\|\rho u\|_{L^{2-r}}, \quad 1 \geq 2 r \geq 0, \\
& \left\|R_{i, j}\left(\rho u_{i} u_{j}\right)\right\|_{L^{2 m}} \lesssim m\|\rho u \otimes u\|_{L^{2 m}}, \quad 1 \leq m<+\infty .
\end{aligned}
$$

Here we have roughly written $\xi=(\Delta)^{-1} \operatorname{div}(\rho u)$ and $\eta=R_{i, j}\left(\rho u_{i} u_{j}\right)$ (with the summation notation).

By Hölder's inequalities we obtain:

$$
\begin{aligned}
& \left\|\nabla(\Delta)^{-1} \operatorname{div}(\rho u)\right\|_{L^{2 m}} \lesssim m\|\rho\|_{L^{\frac{2 m k}{k-1}}}\|u\|_{L^{2 m k}}, \\
& \left\|\nabla(\Delta)^{-1} \operatorname{div}(\rho u)\right\|_{L^{2-r}} \lesssim\|\rho\|_{L^{\frac{2-r}{r}}}\|\sqrt{\rho} u\|_{L^{2}}^{\frac{1}{2}} \\
& \left\|R_{i, j}\left(\rho u_{i} u_{j}\right)\right\|_{L^{2 m}} \lesssim m\|\rho\|_{L^{\frac{2 m k}{k-1}}}\|u\|_{L^{4 m k}}^{2}
\end{aligned}
$$

where $k>1, m \geq 1$ and $r \geq 1,1 \geq 2 r \geq 0$.

From the estimate of lemma 1-3, we obtain:

$$
\begin{aligned}
& \|u\|_{L^{2 m}} \lesssim m^{\frac{1}{2}}\|\nabla u\|_{L^{2}}, \quad m>2, \\
& \left\|(\Delta)^{-1} \operatorname{div}(\rho u)\right\|_{L^{2 m}} \lesssim m^{\frac{1}{2}}\left\|\nabla(\Delta)^{-1} \operatorname{div}(\rho u)\right\|_{\frac{2 m}{m+1}}, \quad m>2 .
\end{aligned}
$$

We set now:

$$
\varphi(t)=\int_{\Omega}\left(\operatorname{curl} u^{2}(t, x)+(2+\lambda(\rho)) \operatorname{div} u^{2}(t, x)\right) d x,
$$

we obtain then by using (5.20), (5.19) with $r=\frac{2}{m+1}$ and the energy inequality (4.16):

$$
\left\|(\Delta)^{-1} \operatorname{div}(\rho u)\right\|_{L^{2 m}} \lesssim m^{\frac{1}{2}}\|\rho\|_{L^{m}}^{\frac{1}{2}}, \quad m>2 .
$$

Similarly we have:

$$
\begin{aligned}
& \left\|\nabla(\Delta)^{-1} \operatorname{div}(\rho u)\right\|_{L^{2 m}} \lesssim m^{\frac{3}{2}} k^{\frac{1}{2}}(\varphi(t))^{\frac{1}{2}}\|\rho\|_{L^{\frac{2 m k}{k-1}}}, \quad m>2, \quad k>1, \\
& \left\|R_{i, j}\left(\rho u_{i} u_{j}\right)\right\|_{L^{2 m}} \lesssim m^{2} k \varphi(t)\|\rho\|_{L^{\frac{2 m k}{k-1}}}, \quad m>2, \quad k>1 .
\end{aligned}
$$


5.1. Gain of integrability for the density. Following [34] the plan of the proof of [34], we are interested in getting a gain of integrability on the density. We follow here the method of Lions in [26] to get a gain of integrability on the pressure and the argument developed in [34]. Apply the operator $(\Delta)^{-1}$ div to the momentum equation, we obtain:

$$
\begin{aligned}
\frac{\partial}{\partial t}(\Delta)^{-1} \operatorname{div}(\rho u) & +\left[R_{i j}, u_{j}\right]\left(\rho u_{i}\right)-(2+\lambda(\rho)) \operatorname{div} u \\
& +P(\rho)-\frac{1}{|\Omega|} \int_{\Omega}(P(\rho)(t, x)-(2+\lambda(\rho)) \operatorname{div} u) d x=0 .
\end{aligned}
$$

We will set in the sequel:

$$
B=(2+\lambda(\rho)) \operatorname{div} u-P(\rho) .
$$

Next if we renormalize the mass equation we have:

$$
\partial_{t} \theta(\rho)+u \cdot \nabla \theta(\rho)+\rho \theta^{\prime}(\rho) \operatorname{div} u=0 .
$$

where we have set:

$$
\theta(\rho)=\int_{1}^{\rho} \frac{1}{s}(2+\lambda(s)) d s=2 \ln \rho+\frac{1}{\beta}\left(\rho^{\beta}-1\right) .
$$

Finally we get the following transport equation:

$$
\begin{aligned}
\frac{\partial}{\partial t}\left[(\Delta)^{-1} \operatorname{div}(\rho u)\right. & +\theta(\rho)]+u \cdot \nabla\left[(\Delta)^{-1} \operatorname{div}(\rho u)+\theta(\rho)\right]+\left[R_{i j}, u_{j}\right]\left(\rho u_{i}\right) \\
& +P(\rho)-\frac{1}{|\Omega|} \int_{\Omega}[P(\rho)(t, x)-(2+\lambda(\rho)) \operatorname{div} u] d x=0
\end{aligned}
$$

Denote by $f$ the function:

$$
f(t, x)=\max \left(0,(\Delta)^{-1} \operatorname{div}(\rho u)+\theta(\rho)\right)
$$

and multiply the equation (5.25) by the function $\rho f^{2 m-1}$ with $m \in \mathbb{N}$ and $m \geq 4$ and integrate over $\Omega$, we obtain:

$$
\begin{aligned}
\frac{1}{2 m} \frac{d}{d t} \int_{\Omega} \rho f^{2 m} d x+\int_{\Omega} \rho P(\rho) f^{2 m-1} d x & +\int_{\Omega}\left[R_{i j}, u_{j}\right]\left(\rho u_{i}\right) \rho f^{2 m-1} d x \\
& +\int_{\Omega} B d x \int_{\Omega} \rho f^{2 m-1} d x=0 .
\end{aligned}
$$

As in [34] we set:

$$
Z(t)=\left(\int_{\Omega} \rho f^{2 m}(t, x)\right)^{\frac{1}{2 m}}
$$

Using Hölder's inequality $\left(\frac{2 m-1}{2 m}+\frac{1}{2 m}=1, \frac{\beta}{2 m \beta+1}+\frac{1}{2 m(2 m \beta+1)}=\frac{1}{2 m}\right)$, we begin with estimating the term $\mid \int_{\Omega}\left[R_{i j}, u_{j}\right]\left(\rho u_{i}\right) \rho f^{2 m-1} d x$ in (5.26) as follows:

$$
\begin{aligned}
\left|\int_{\Omega}\left[R_{i j}, u_{j}\right]\left(\rho u_{i}\right) \rho f^{2 m-1} d x\right| & \leq \int_{\Omega}\left|\left[R_{i j}, u_{j}\right]\left(\rho u_{i}\right)\right| \rho^{\frac{1}{2 m}} \rho^{\frac{2 m-1}{2 m}} f^{2 m-1} d x \\
& \leq\left\|\left|\left[R_{i j}, u_{j}\right]\left(\rho u_{i}\right)\right| \rho^{\frac{1}{2 m}}\right\|_{L^{2 m}(\Omega)} Z^{2 m-1}(t) \\
& \leq\|\rho\|_{L^{2 m} \frac{1}{2 m}}\left\|\left[R_{i j}, u_{j}\right]\left(\rho u_{i}\right)\right\|_{L^{2 m+\frac{1}{\beta}}(\Omega)} Z^{2 m-1}(t)
\end{aligned}
$$


Next we recall a result of R. Coifman, P.-L. Lions, Y. Meyer and S. Semmes in [7], which says that the following map:

$$
\begin{aligned}
W^{1, r_{1}}\left(\mathbb{T}^{N}\right)^{N} \times L^{r_{2}}\left(\mathbb{T}^{N}\right)^{N} & \rightarrow W^{1, r_{3}}\left(\mathbb{T}^{N}\right)^{N} \\
(a, b) & \rightarrow\left[a_{j}, R_{i, j}\right] b_{i}
\end{aligned}
$$

is continuous for any $N \geq 2$ as soon as $\frac{1}{r_{3}}=\frac{1}{r_{1}}+\frac{1}{r_{2}}$. Hence we obtain that $\left[R_{i j}, u_{j}\right]\left(\rho u_{i}\right)$ belongs in $W^{1, p}$ (where $\frac{1}{p}=\frac{1}{2}+\frac{1}{2\left(m+\frac{1}{2 \beta}\right) k}+\frac{k-1}{2\left(m+\frac{1}{2 \beta}\right) k}$ with $k>1$ and $\left.p=2-\frac{2}{m+1+\frac{1}{2 \beta}}<2\right)$ with the following inequality:

$$
\begin{aligned}
\left\|\left[R_{i j}, u_{j}\right]\left(\rho u_{i}\right)\right\|_{W^{1, p}(\Omega)} & \leq C\|\nabla u\|_{L^{2}(\Omega)}\|u\|_{L^{2\left(m+\frac{1}{2 \beta}\right) k}(\Omega)}\|\rho\|_{L^{\frac{2\left(m+\frac{1}{2 j}\right) k}{k-1}}(\Omega)}, \\
& \leq C\|\nabla u\|_{L^{2}(\Omega)}\|u\|_{L^{2\left(m+\frac{1}{2 \beta}\right) k}(\Omega)}\|\rho\|_{L^{2 m \beta+1}(\Omega)},
\end{aligned}
$$

where we have choose $k$ such that $\frac{2\left(m+\frac{1}{2 \beta}\right) k}{k-1}=2 m \beta+1$, let $k=\frac{2 m \beta+1}{2 m(\beta-1)+1-\frac{1}{\beta}}$. We verifies that $\frac{1}{q}=\frac{1}{p}-\frac{1}{2}=\frac{1}{2 m+\frac{1}{\beta}}$. Next by using lemma 2 and (5.29) we get:

$$
\begin{aligned}
& \left\|\left[R_{i j}, u_{j}\right]\left(\rho u_{i}\right)(t, \cdot)\right\|_{L^{2 m+\frac{1}{\beta}}(\Omega)} \\
& \begin{array}{r}
\lesssim\left(m^{\frac{1}{2}}\|\nabla u(t, \cdot)\|_{L^{2}(\Omega)}\|u(t, \cdot)\|_{L^{2\left(m+\frac{1}{2 \beta}\right) k}(\Omega)}\|\rho(t, \cdot)\|_{L^{2 m \beta+1}(\Omega)}\right. \\
\left.+\left|\int_{\Omega}\left[R_{i j}, u_{j}\right]\left(\rho u_{i}\right)(t, x) d x\right|\right) .
\end{array}
\end{aligned}
$$

We can easily bound the last term on the right hand side by using the continuity of the Riez transform in $L^{p}(\omega)$ with $1<p<+\infty$ :

$$
\left|\int_{\Omega}\left[R_{i j}, u_{j}\right]\left(\rho u_{i}\right)(t, x) d x\right| \lesssim\|\rho(t, \cdot)\|_{L^{\gamma}(\Omega)}\|u(t, \cdot)\|_{H^{1}(\Omega)}^{2} .
$$

By (5.20) and the previous inequalities we obtain finally:

$$
\begin{aligned}
& \left\|\left[R_{i j}, u_{j}\right]\left(\rho u_{i}\right)(t, \cdot)\right\|_{L^{2 m+\frac{1}{\beta}}(\Omega)} \\
& \leq C m\|\nabla u\|_{L^{2}(\Omega)}^{2}\|\rho\|_{L^{2 m \beta+1}(\Omega)}+\|\rho(t, \cdot)\|_{L^{\gamma}(\Omega)}\|u(t, \cdot)\|_{H^{1}(\Omega)}^{2} .
\end{aligned}
$$

We have then from (5.28) and (5.30):

$$
\begin{aligned}
& \left|\int_{\Omega}\left[R_{i j}, u_{j}\right]\left(\rho u_{i}\right) \rho f^{2 m-1} d x\right| \\
& \lesssim\left(m\|\rho\|_{L^{2 m \beta+1}(\Omega)}^{1+\frac{1}{2 m}} \varphi(t)+\|\rho(t, \cdot)\|_{L^{\gamma}(\Omega)}\|u(t, \cdot)\|_{H^{1}(\Omega)}^{2}\right) Z^{2 m-1}(t) .
\end{aligned}
$$

Next as in [34] we get:

$$
\begin{aligned}
\left|\int_{\Omega} B d x \int_{\Omega} \rho f^{2 m-1} d x\right| & \lesssim Z^{2 m-1}(t)\|\rho\|_{L^{1}}^{\frac{1}{2 m}} \int_{\Omega}((2+\lambda)|\operatorname{div} u|+P) d x \\
& \lesssim Z^{2 m-1}(t)\left(1+(\varphi(t))^{\frac{1}{2}}\left(\int_{\Omega}(2+\lambda(\rho)) d x\right)^{\frac{1}{2}}\right) \\
& \lesssim Z^{2 m-1}(t)\left(1+\varphi(t)^{\frac{1}{2}}+\|\rho\|_{L^{2 m \beta+1}(\Omega)}^{\frac{\beta}{2}} \varphi(t)^{\frac{1}{2}}\right) .
\end{aligned}
$$


Collecting all the above inequalities, we obtain:

$$
\begin{aligned}
Z(t) \lesssim 1+\int_{0}^{t}\|\rho\|_{L^{\gamma}(\Omega)}(\tau)\|u\|_{H^{1}(\Omega)}^{2}(\tau) d \tau & +\int_{0}^{t} m \varphi(\tau)\|\rho\|_{L^{2 m \beta+1}(\Omega)}^{1+\frac{1}{2 m}}(\tau) d \tau \\
& +\int_{0}^{t} \varphi(\tau)^{\frac{1}{2}}\|\rho\|_{L^{2 m \beta+1}(\Omega)}^{\frac{\beta}{2}}(\tau) d \tau .
\end{aligned}
$$

As we have seen that $u$ belongs in $L^{2}\left((0, t), H^{1}(\Omega)\right)$ we have:

$$
\int_{0}^{t}\|\rho\|_{L^{\gamma}(\Omega)}(\tau)\|u\|_{H^{1}(\Omega)}^{2}(\tau) d \tau \lesssim 1
$$

We obtain then:

$$
Z(t) \lesssim 1+\int_{0}^{t} m \varphi(\tau)\|\rho\|_{L^{2 m \beta+1}(\Omega)}^{1+\frac{1}{2 m}}(\tau) d \tau+\int_{0}^{t} \varphi(\tau)^{\frac{1}{2}}\|\rho\|_{L^{2 m \beta+1}(\Omega)}^{\frac{\beta}{2}}(\tau) d \tau .
$$

Next following [34] we introduce the measurable sets:

$$
\Omega_{1}(t)=\left\{x \in \Omega / \rho \geq 2 m^{\prime}\right\} \text { and } \Omega_{2}(t)=\left\{x \in \Omega_{1}(t) / \theta(\rho)+(\Delta)^{-1} \operatorname{div}(\rho u)>0\right\} \text {. }
$$

We then have:

$$
\|\rho\|_{L^{2 m \beta+1}(\Omega)}^{\beta} \lesssim\left(\int_{\Omega_{1}(t)} \rho^{2 m \beta+1} d x\right)^{\frac{\beta}{2 m \beta+1}}+1
$$

Moreover by the definition of the function $\theta(\rho)$, we have:

$$
\left(\int_{\Omega_{1}(t)} \rho^{2 m \beta+1} d x\right)^{\frac{\beta}{2 m \beta+1}} \lesssim\left(\int_{\Omega_{1}(t)} \rho \theta(\rho)^{2 m} d x\right)^{\frac{\beta}{2 m \beta+1}}
$$

Using the fact that on $\Omega_{1}(t) \backslash \Omega_{2}(t)$ we have $0 \leq \theta(\rho) \leq\left|(\Delta)^{-1} \operatorname{div}(\rho u)\right|$, we derive the following estimate:

$$
\begin{aligned}
& \int_{\Omega_{1}(t)} \rho \theta(\rho)^{2 m} d x \\
& =\int_{\Omega_{2}(t)} \rho\left(\theta(\rho)+(\Delta)^{-1} \operatorname{div}(\rho u)-(\Delta)^{-1} \operatorname{div}(\rho u)\right)^{2 m} d x+\int_{\Omega_{1}(t) \backslash \Omega_{2}(t)} \rho \theta(\rho)^{2 m} d x \\
& \leq 2^{2 m-1}\left(\int_{\Omega_{2}(t)} \rho f(\rho)^{2 m} d x+\int_{\Omega_{2}(t)} \rho\left|(\Delta)^{-1} \operatorname{div}(\rho u)\right|^{2 m} d x\right) \\
& \quad+\int_{\Omega_{1}(t) \backslash \Omega_{2}(t)} \rho\left|(\Delta)^{-1} \operatorname{div}(\rho u)\right|^{2 m} d x \\
& \leq 2^{2 m}\left(Z^{2 m}(t)+\int_{\Omega} \rho\left|(\Delta)^{-1} \operatorname{div}(\rho u)\right|^{2 m} d x\right) .
\end{aligned}
$$

From estimates (5.33) and (5.34) we deduce:

$$
\|\rho\|_{L^{2 m \beta+1}(\Omega)}^{\beta} \leq C\left(\left(m^{\prime}\right)^{\beta}+Z(t)^{\frac{2 m \beta}{2 m \beta+1}}(t)+\left(\int_{\Omega} \rho\left|(\Delta)^{-1} \operatorname{div}(\rho u)\right|^{2 m} d x\right)^{\frac{\beta}{2 m \beta+1}}\right) .
$$


In view of estimate $(5.21),(5.22)$, we have:

$$
\begin{aligned}
\int_{\Omega} \rho\left|(\Delta)^{-1} \operatorname{div}(\rho u)\right|^{2 m} d x & \leq\|\rho\|_{L^{2 m \beta+1}(\Omega)}\left\|(\Delta)^{-1} \operatorname{div}(\rho u)\right\|_{L^{2 m+\frac{1}{\beta}}(\Omega)}^{2 m} \\
& \leq\|\rho\|_{L^{2 m \beta+1}(\Omega)}\left(\left(m+\frac{1}{2 \beta}\right)^{\frac{1}{2}}\|\rho\|_{L^{m+\frac{1}{\beta}}(\Omega)}^{\frac{1}{2}}\right)^{2 m} \\
& \leq C^{m} m^{m}\|\rho\|_{L^{2 m \beta+1}(\Omega)}^{m+1}
\end{aligned}
$$

Finally:

$$
\|\rho\|_{L^{2 m \beta+1}(\Omega)}^{\beta} \lesssim\left(Z(t)^{\frac{2 m \beta}{2 m \beta+1}}(t)+m^{\frac{1}{2}}\|\rho\|_{L^{2 m \beta+1}(\Omega)}^{\frac{\beta(m+1)}{2 m \beta+1}}\right)
$$

By Young's inequality (with $q=\frac{2 m \beta+1}{m+1}$ and $p=\frac{2 \beta}{2 \beta-1}+\frac{1}{m(2 \beta-1)}$ ), we obtain:

$$
\|\rho\|_{L^{2 m \beta+1}(\Omega)}^{\beta} \lesssim\left(Z(t)+\frac{1}{\varepsilon} m^{\frac{\beta}{2 \beta-1}+\frac{1}{2 m(2 \beta-1)}}+\varepsilon\|\rho\|_{L^{2 m \beta+1}(\Omega)}^{\beta}\right) .
$$

By bootstrap, we get:

$$
\|\rho\|_{L^{2 m \beta+1}(\Omega)}^{\beta} \lesssim Z(t)+m^{\frac{\beta}{2 \beta-1}} .
$$

Therefore (5.31) and (5.36)give the following inequality:

$\|\rho\|_{L^{2 m \beta+1}(\Omega)}^{\beta} \lesssim\left(m^{\frac{\beta}{2 \beta-1}}+\int_{0}^{t} m \varphi(\tau)\|\rho\|_{L^{2 m \beta+1}(\Omega)}^{1+\frac{1}{2 m}}(\tau) d \tau+\int_{0}^{t} \varphi(\tau)^{\frac{1}{2}}\|\rho\|_{L^{2 m \beta+1}(\Omega)}^{\frac{\beta}{2}}(\tau) d \tau\right)$.

Next by Young's inequality, we have:

$\|\rho\|_{L^{2 m \beta+1}(\Omega)}^{\beta} \lesssim\left(1+m^{\frac{\beta}{2 \beta-1}}+\int_{0}^{t} m \varphi(\tau)\|\rho\|_{L^{2 m \beta+1}(\Omega)}^{1+\frac{1}{2 m}}(\tau) d \tau+\int_{0}^{t}\|\rho\|_{L^{2 m \beta+1}(\Omega)}^{\beta}(\tau) d \tau\right)$.

Using the fact that $\varphi(t) \in L^{1}(0, T)$ and applying Grönwall's inequality, wehave that:

$$
\|\rho\|_{L^{2 m \beta+1}(\Omega)}^{\beta} \leq C\left(1+m^{\frac{\beta}{2 \beta-1}}+\int_{0}^{t} m \varphi(\tau)\|\rho\|_{L^{2 m \beta+1}(\Omega)}^{1+\frac{1}{2 m}}(\tau) d \tau,\right)
$$

where $C$ depends on $t$. Denote:

$$
y(t)=m^{-\frac{1}{\beta-1}}\|\rho\|_{L^{2 m \beta+1}(\Omega)}, \quad t \in[0, T] .
$$

Then:

$$
y^{\beta}(t) m^{\frac{\beta}{\beta-1}} \leq C\left(1+m^{\frac{\beta}{2 \beta-1}}+m m^{\left(1+\frac{1}{2 m}\right) \frac{1}{\beta-1}} \int_{0}^{t} \varphi(\tau) y^{1+\frac{1}{2 m}}(\tau) d \tau\right)
$$

and we have:

$$
m m^{\left(1+\frac{1}{2 m}\right) \frac{1}{\beta-1}}=m^{\frac{\beta}{\beta-1}+\frac{1}{2 m(\beta-1)}} .
$$

We have then:

$$
y^{\beta}(t) \leq C\left(1+m^{\frac{-\beta^{2}}{(\beta-1)(2 \beta-1)}}+m^{\frac{1}{2 m(\beta-1)}} \int_{0}^{t} \varphi(\tau) y^{1+\frac{1}{2 m}}(\tau) d \tau\right)
$$


where $\frac{\beta}{2 \beta-1}-\frac{\beta}{\beta-1}=\frac{-\beta^{2}}{(\beta-1)(2 \beta-1)}<0$.

Recalling that $\beta>1$ and $\varphi(t) \in L^{1}(0, T)$ we find that for $m$ big enough:

$$
y^{\beta}(t) \leq C\left(C_{1}+\int_{0}^{t} \varphi(\tau) y^{\beta}(\tau) d \tau\right)
$$

whence by Grönwall inequality:

$$
y(t) \leq C, \quad t \in[0, T],
$$

where $C$ depends on $t$. We thus have:

$$
\|\rho\|_{L^{2 m \beta+1}} \leq C m^{\frac{1}{\beta-1}}, \quad t \in[0, T] .
$$

Hence the inequality:

$$
\|\rho\|_{L^{k}(\Omega)}(t) \leq C k^{\frac{1}{\beta-1}}, \quad t \in[0, T] .
$$

is valid for every $k \geq 1$, with $C$ a positive constant independent of $k \geq 1$ but depending of the time.

REMARK 6. Let us point out that the estimate (5.38) is the key point in order to improve the range on $\beta$. Indeed this last one is a refinement of the corresponding one in [34]. In particular we will be able to obtain the energy estimates (5.66) only with assuming $\beta>2$.

5.2. Second a priori estimate for the velocity. In this section, we are going to furnish estimates on the velocity by using the gain of integrability on the density proved in the previous section. We are going essentially to follow the proof of [34] and to emphasize on the key point where we will only need the hypothesis $\beta>2$. We begin with recalling some equation on the effective pressure defined in [26] and the rotational curl. We set:

$$
\begin{aligned}
& A=\operatorname{curl} u \text { and } B=(2+\lambda(\rho)) \operatorname{div} u-P(\rho), \\
& L=\frac{1}{\rho}\left(\partial_{y} A+\partial_{x} B\right) \text { and } H=\frac{1}{\rho}\left(-\partial_{x} A+\partial_{y} B\right) .
\end{aligned}
$$

We now want to obtain some estimates on the unknowns $A$ and $B$, let us start with rewriting the momentum equation under the following eulerian form:

$$
\partial_{t} u+u \cdot \nabla u-\frac{1}{\rho} \Delta u-\frac{1}{\rho} \nabla((\mu+\lambda(\rho)) \operatorname{div} u)+\nabla\left(\frac{P(\rho)}{\gamma \rho}\right)=0 .
$$

Next if we apply the operator curl, we get:

$$
\partial_{t} A+u \cdot \nabla A+A \operatorname{div} u=\partial_{y} L-\partial_{x} H .
$$

Next we apply the operator div to the momentum equation (5.39):

$$
\partial_{t} \operatorname{div} u+u \cdot \nabla u-\frac{1}{\rho} \Delta u-\frac{1}{\rho} \nabla((\mu+\lambda(\rho)) \operatorname{div} u)+\nabla\left(\frac{P(\rho)}{\gamma \rho}\right)=0,
$$

and via the mass equation we have:

$$
\begin{aligned}
\partial_{t} B+U \cdot \nabla B & -\rho(2+\lambda)\left(B\left(\frac{1}{2+\lambda}\right)^{\prime}+\left(\frac{P}{2+\lambda}\right)^{\prime}\right) \operatorname{div} u \\
& +(2+\lambda)\left(U_{x}^{2}+2 U_{y} V_{x}+V_{y}^{2}\right)=(2+\lambda)\left(L_{x}+H_{y}\right) .
\end{aligned}
$$


As in [34] multiplying the equation(5.40) by $A$ and integrate over $\Omega$ we obtain:

$$
\int_{\Omega} \frac{1}{2} \frac{d}{d t}\left[A^{2}\right] d x+\frac{1}{2} \int_{\Omega} \operatorname{div} u A^{2} d x+\int_{\Omega}\left(L \partial_{y} A-H \partial_{x} A\right) d x=0 .
$$

Similarly multiplying the equation $(5.42)$ by $\frac{1}{2+\lambda} B$ and integrate over $\Omega$ we have:

$$
\begin{aligned}
\int_{\Omega} \frac{1}{2+\lambda} \frac{d}{d t}\left(\frac{1}{2} B^{2}\right) d x-\frac{1}{2} \int_{\Omega} \operatorname{div} u \frac{B^{2}}{2+\lambda} d x-\frac{1}{2} \int_{\Omega} u \cdot \nabla\left(\frac{1}{2+\lambda}\right) B^{2} d x \\
-\int_{\Omega} \rho B \operatorname{div} u\left(B\left(\frac{1}{2+\lambda}\right)^{\prime}+\left(\frac{P}{2+\lambda}\right)^{\prime}\right) d x+\int_{\Omega} B\left(U_{x}^{2}+2 U_{y} V_{x}+V_{y}^{2}\right) d x \\
+\int_{\Omega}\left(L \partial_{x} B+H \partial_{y} B\right) d x=0 .
\end{aligned}
$$

We recall now that:

$$
\partial_{t}\left(\frac{1}{2+\lambda}\right)+\left(\frac{1}{2+\lambda}\right)^{\prime} \rho \operatorname{div} u+\nabla\left(\frac{1}{2+\lambda}\right) \cdot u=0
$$

By combining the previous equality and (5.44) we get:

$$
\begin{aligned}
& \frac{1}{2} \int_{\Omega} \frac{d}{d t}\left(\frac{1}{2+\lambda} B^{2}\right) d x-\frac{1}{2} \int_{\Omega} \operatorname{div} u B^{2}\left(\frac{1}{2+\lambda}-\rho\left(\frac{1}{2+\lambda}\right)^{\prime}\right) d x \\
& -\int_{\Omega} \rho B \operatorname{div} u\left(B\left(\frac{1}{2+\lambda}\right)^{\prime}+\left(\frac{P}{2+\lambda}\right)^{\prime}\right) d x+\int_{\Omega} B(\operatorname{div} u)^{2} d x \\
& +2 \int_{\Omega} B\left(\partial_{y} U \partial_{x} V-\partial_{x} U \partial_{y} V\right) d x+\int_{\Omega}\left(L \partial_{x} B+H \partial_{y} B\right) d x=0
\end{aligned}
$$

Summing (5.43) and (5.45) we have:

$$
\begin{aligned}
& \int_{\Omega} \frac{1}{2} \frac{d}{d t}\left[\left(A^{2}+\frac{B^{2}}{2+\lambda}\right)\right] d x+\int_{\Omega} \frac{1}{2} \operatorname{div} u A^{2} d x+\int_{\Omega} \frac{\left(A_{y}+B_{x}\right)^{2}+\left(-A_{x}+B_{y}\right)^{2}}{\rho} d x \\
& -\frac{1}{2} \int_{\Omega} B^{2} \operatorname{div} u\left(\frac{1}{2+\lambda}-\rho\left(\frac{1}{2+\lambda}\right)^{\prime}\right) d x+2 \int_{\Omega} B\left(U_{y} V_{x}-U_{x} V_{y}\right) d x \\
& -\int_{\Omega} \rho B \operatorname{div} u\left(B\left(\frac{1}{2+\lambda}\right)^{\prime}+\left(\frac{P}{2+\lambda}\right)^{\prime}\right) d x+\int_{\Omega} B \operatorname{div} u^{2} d x=0 .
\end{aligned}
$$

As:

$$
\operatorname{div} u^{2}=\operatorname{div} u\left(\frac{B}{2+\lambda}+\frac{P}{2+\lambda}\right),
$$

we deduce:

$$
\begin{aligned}
& \int_{\Omega} \frac{1}{2} \frac{d}{d t}\left[\left(A^{2}+\frac{B^{2}}{2+\lambda}\right)\right] d x+\int_{\Omega} \frac{1}{2} \operatorname{div} u A^{2} d x+\int_{\Omega} \frac{\left(A_{y}+B_{x}\right)^{2}+\left(-A_{x}+B_{y}\right)^{2}}{\rho} d x \\
& \left.\left.+\int_{\Omega} \frac{1}{2} B^{2} \operatorname{div} u\left(\frac{1}{2+\lambda}-\rho\left(\frac{1}{2+\lambda}\right)^{\prime}\right) d x+\int_{\Omega} B \operatorname{div} u\left(\frac{P}{2+\lambda}\right)-\rho\left(\frac{P}{2+\lambda}\right)\right)^{\prime}\right) d x \\
& +2 \int_{\Omega} B\left(U_{y} V_{x}-U_{x} V_{y}\right) d x-\int_{\Omega} \rho B \operatorname{div} u\left(B\left(\frac{1}{2+\lambda}\right)^{\prime}+\left(\frac{P}{2+\lambda}\right)^{\prime}\right) d x=0 .
\end{aligned}
$$


Let us set:

$$
\begin{aligned}
& Z(t)=\left(\int_{\Omega}\left(A^{2}+\frac{B^{2}}{2+\lambda}\right) d x\right)^{\frac{1}{2}} \\
& a(t)=\left(\int_{\Omega} \frac{\left(A_{y}+B_{x}\right)^{2}+\left(-A_{x}+B_{y}\right)^{2}}{\rho} d x\right)^{\frac{1}{2}}, \quad t \in[0, T] .
\end{aligned}
$$

Next we have:

$$
\int_{\Omega}\left(\left(A_{y}+B_{x}\right)^{2}+\left(-A_{x}+B_{y}\right)^{2}\right) d x=\int_{\Omega}\left(A_{x}^{2}+A_{y}^{2}+B_{x}^{2}+B_{y}^{2}\right) d x .
$$

Let us observe that for every $r, 1 \geq 4 r>0$, from the result on elliptic system and by Hölder inequalities we get as in [34]:

$$
\begin{array}{r}
\|\nabla A\|_{L^{2(1-r)}(\Omega)}+\|\nabla B\|_{L^{2(1-r)}(\Omega)} \leq C\left(\int_{\Omega} \frac{\left(A_{y}+B_{x}\right)^{2}+\left(-A_{x}+B_{y}\right)^{2}}{\rho} d x\right)^{\frac{1}{2}} \\
\left(\int_{\Omega} \rho^{\frac{1-r}{r}} d x\right)^{\frac{r}{2(1-r)}} .
\end{array}
$$

From (5.38), we have:

$$
\left(\|\nabla A\|_{L^{2(1-r)}(\Omega)}+\|\nabla B\|_{L^{2(1-r)}(\Omega)}\right) \leq C\left(\frac{1}{r}\right)^{\frac{1}{2(\beta-1)}} a(t) .
$$

REMARK 7. Let us point out that the estimate (5.48) is better than the corresponding one in [34] due to the better estimate (5.38).

Moreover via (5.38) we also obtain the following inequality:

$$
\left(\|\nabla u\|_{L^{2}(\Omega)}+\|A\|_{L^{2}(\Omega)}+\|\sqrt{2+\lambda} \operatorname{div} u\|_{L^{2}(\Omega)}\right) \leq C(1+Z(t)), \quad t \in[0, T] .
$$

Now, are interested in providing other estimates for the non positive terms of the equality (6.76).

Estimates for the terms of (6.76). Following [34], using the lemma 1 (with $\alpha=\frac{1-\varepsilon}{2(1-2 \varepsilon)}$ ) and Young's inequality (with $p=\frac{2(1-2 \varepsilon)}{1-\varepsilon}, q=\frac{2(1-2 \varepsilon)}{1-3 \varepsilon}$ and $\left.p_{1}=\frac{(1-2 \varepsilon)(2+\varepsilon)}{1-\varepsilon}, q_{1}=\frac{(1-2 \varepsilon)(2+\varepsilon)}{1-2 \varepsilon-2 \varepsilon^{2}}\right)$ the, we obtain:

$$
\begin{aligned}
\left|\frac{1}{2} \int_{\Omega} \operatorname{div} u A^{2}\right| & \leq \frac{1}{2}\|\operatorname{div} u(t)\|_{L^{2}}\|A(t)\|_{L^{4}}^{2} \\
& \leq C\|\operatorname{div} u\|_{L^{2}}\left(\frac{1}{2}\|A\|_{L^{2}}\right)^{\frac{1-3 \varepsilon}{1-2 \varepsilon}}\left(\frac{1}{2}\|\nabla A\|_{L^{2(1-\varepsilon)}}\right)^{\frac{1-\varepsilon}{1-2 \varepsilon}} \\
& \leq C(1+Z(t)) Z(t)^{\frac{1-3 \varepsilon}{1-2 \varepsilon}}\left(\left(\frac{1}{\varepsilon}\right)^{\frac{1}{2(\beta-1)}} a(t)\right)^{\frac{1-\varepsilon}{1-2 \varepsilon}} \\
& \leq \delta a^{2}(t)+C(\delta)(1+Z(t))^{\frac{2(1-2 \varepsilon)}{1-3 \varepsilon}} Z(t)^{2}\left(\frac{1}{\varepsilon}\right)^{\frac{1}{\beta-1}} \\
& \leq \delta a^{2}(t)+C(\delta)\left(1+Z(t)^{2}\right)^{2+\frac{\varepsilon}{1-3 \varepsilon}}\left(\frac{1}{\varepsilon}\right)^{\frac{1}{\beta-1}}
\end{aligned}
$$

We now are interested in estimating the term in (6.76) corresponding to:

$$
\begin{aligned}
I_{1} & =\left|\frac{1}{2} \int_{\Omega} B^{2} \operatorname{div} u\left(\frac{1}{2+\lambda}-\rho\left(\frac{1}{2+\lambda}\right)^{\prime}\right) d x\right| \\
& =\left|\frac{1}{2} \int_{\Omega} B^{2}\left(\frac{B}{2+\lambda}+\frac{P}{2+\lambda}\right)\left(\frac{1}{2+\lambda}-\rho\left(\frac{1}{2+\lambda}\right)^{\prime}\right) d x\right| .
\end{aligned}
$$


Easily there exist a positive constant $C>0$ such that:

$$
\left|\frac{1}{2+\lambda}-\rho\left(\frac{1}{2+\lambda}\right)^{\prime}\right| \leq C
$$

for all $\rho \in[0,+\infty)$. We deduce that:

$$
I_{1} \leq C\left(m^{\prime}\right)^{\beta}\left(\int_{\Omega} \frac{|B|^{3}}{2+\lambda} d x+\int_{\Omega} \frac{|B|^{2}}{2+\lambda}|P| d x\right) .
$$

By Young's inequality we have:

$$
\begin{aligned}
\left|\int_{\Omega} B \operatorname{div} u\left(\frac{P}{2+\lambda}-\rho\left(\frac{P}{2+\lambda}\right)^{\prime}\right) d x\right| & =\left|\int_{\Omega} B\left(\frac{B}{2+\lambda}+\frac{P}{2+\lambda}\right)\left(\frac{P}{2+\lambda}-\rho\left(\frac{P}{2+\lambda}\right)^{\prime}\right) d x\right| \\
& \leq C\left(1+\int_{\Omega} \frac{|B|^{3}}{2+\lambda} d x\right) .
\end{aligned}
$$

Now, the last term in (6.76) can be treated as follows:

$$
\left|2 \int_{\Omega} B\left(U_{y} V_{x}-U_{x} V_{y}\right) d x\right| \leq \int_{\Omega}|B|\left(U_{x}^{2}+U_{y}^{2}+V_{x}^{2}+V_{y}^{2}\right) d x .
$$

Via the previous estimate, the notations (5.47), and using the equality (6.76), we get:

$$
\begin{aligned}
\frac{1}{2}\left(Z^{2}(t)\right)+a^{2}(t) \leq & \delta a^{2}(t)+C(\delta)\left(1+Z(t)^{2}\right)^{1+\frac{\varepsilon}{1-3 \varepsilon}}\left(\frac{1}{\varepsilon}\right)^{\frac{2}{\beta-1}} \\
& +C\left(1+\int_{\Omega} \frac{|B|^{3}}{2+\lambda} d x\right)+\int_{\Omega}|B|\left(U_{x}^{2}+U_{y}^{2}+V_{x}^{2}+V_{y}^{2}\right) d x
\end{aligned}
$$

It remains to estimate the two last terms on the right hand side of (5.50). In this goal, from (2.10) we have:

$$
\|B\|_{L^{2 m}(\Omega)} \leq C\left(\|B\|_{L^{1}(\Omega)}+m^{\frac{1}{2}}\|\nabla B\|_{L^{\frac{2 m}{m+\varepsilon}}(\Omega)}^{1-s}\|B\|_{L^{2(1-\varepsilon)}(\Omega)}^{s}\right)
$$

where: $s=\frac{(1-\varepsilon)^{2}}{m-\varepsilon(1-\varepsilon)}$ and $C>0$ is a positive constant independent of $m>2$.

Now in inequalities (5.50) and (5.51) we fix $\varepsilon=2^{-m}$ with $m>2$. Using estimate (5.38) for the density, we derive the inequalities:

$$
\begin{aligned}
\|B\|_{L^{1}(\Omega)}=\int_{\Omega}|B| d x & =\int_{\Omega}\left(\frac{1}{2+\lambda}\right)^{\frac{1}{2}}|B|(2+\lambda)^{\frac{1}{2}} d x \leq\left\|(2+\lambda)^{\frac{1}{2}}\right\|_{L^{2}(\Omega)} Z(t) \leq C Z(t) . \\
\|B\|_{L^{2(1-\varepsilon)(\Omega)}}^{s} & =\left(\int_{\Omega}\left(\frac{1}{2+\lambda}\right)^{1-\varepsilon}|B|^{2(1-\varepsilon)}(2+\lambda)^{1-\varepsilon} d x\right)^{\frac{s}{2(1-\varepsilon)}} \\
& \leq Z(t)^{s}\|2+\lambda\|^{\frac{s}{2}} L^{\frac{1-\varepsilon}{\varepsilon}}(\Omega) \\
\leq C & \leq C\left(\frac{1}{\varepsilon}\right)^{\frac{\beta s}{2(\beta-1)}} Z^{s}(t) \leq C 2^{2 s m} Z^{s}(t), \\
& \leq C Z^{s}(t)
\end{aligned}
$$

From inequalities (5.48) and (5.51) we finally obtain:

$$
\begin{aligned}
\|B\|_{L^{2 m}(\Omega)} & \leq C\left(Z(t)+m^{\frac{1}{2}}\left(\frac{m}{\varepsilon}\right)^{\frac{1-s}{2(\beta-1)}}(a(t))^{1-s} Z^{s}(t)\right), \\
& \leq C\left(Z(t)+m^{\frac{1}{2}}\left(\frac{m}{\varepsilon}\right)^{\frac{1-s}{2(\beta-1)}}(a(t))^{1-s} Z^{s}(t)\right) .
\end{aligned}
$$


Now dealing with the integral with $|B|^{3}$, we have:

$$
\begin{aligned}
& \int_{\Omega} \frac{|B|^{3}}{2+\lambda} d x=\int_{\Omega} \frac{|B|^{2-\frac{1}{m-1}}}{(2+\lambda)^{1-\frac{1}{2(m-1)}}}\left(\frac{1}{2+\lambda}\right)^{\frac{1}{2(m-1)}}|B|^{1+\frac{1}{m-1}} d x \\
& \leq \int_{\Omega} \frac{|B|^{2-\frac{1}{m-1}}}{(2+\lambda)^{1-\frac{1}{2(m-1)}}}|B|^{\frac{m}{m-1}} d x \leq Z(t)^{2-\frac{1}{m-1}}\|B\|_{L^{2 m}(\Omega)}^{\frac{m}{m-1}}, \\
& \leq\left(\int_{\Omega} \frac{|B|^{2}}{2+\lambda} d x\right)^{1-\frac{1}{2(m-1)}}\left(\int_{\Omega}|B|^{2 m} d x\right)^{\frac{1}{2(m-1)}} \leq^{-1+\frac{1}{2(m-1)}} Z(t)^{2-\frac{1}{m-1}}\|B\|_{L^{2 m}(\Omega)}^{\frac{m}{m-1}} \\
& \leq C Z^{2-\frac{1}{m-1}}(t)\left(Z(t)^{\frac{m}{m-1}}+m^{\frac{m}{2(m-1)}}\left(\frac{m}{\varepsilon}\right)^{\frac{m(1-s)}{2(\beta-1)(m-1)}}(a(t))^{\frac{m(1-s)}{m-1}} Z^{\frac{m s}{m-1}}(t)\right), \\
& \leq C\left(Z(t)^{3}+m^{\frac{m}{2(m-1)}}\left(\frac{m}{\varepsilon}\right)^{\frac{m(1-s)}{2(\beta-1)(m-1)}}(a(t))^{\frac{m(1-s)}{m-1}} Z^{2+\frac{m s-1}{m-1}}(t)\right),
\end{aligned}
$$

where $C>0$ is a positive constant independent of $m>2$ and $\varepsilon=2^{-m}$. Finally applying applying Young's inequality with $p=\frac{2(m-1)}{m(1-s)}$ and $q=\frac{2(m-1)}{m(s+1)-2}$ we have:

$$
\begin{aligned}
& \int_{\Omega} \frac{|B|^{3}}{2+\lambda} d x \leq C\left(Z^{3}(t)+m^{\frac{1}{2}}\left(\frac{m}{\varepsilon}\right)^{\frac{1}{2(\beta-1)}} a^{\frac{m(1-s)}{m-1}}(t) Z^{2+\frac{m s-1}{m-1}}(t)\right) \\
& \leq \delta a^{2}(t)+C(\delta)\left(Z^{3}(t)+m^{\frac{m-1}{m(s+1)-2}}\left(\frac{m}{\varepsilon}\right)^{\frac{m-1}{(\beta-1)(m(s+1)-2)}} Z^{4+\frac{2(1-m s)}{m(s+1)-2}}(t)\right)
\end{aligned}
$$

From (5.51), we verify that:

$$
1-m s=1-\frac{m(1-\varepsilon)^{2}}{m-\varepsilon(1-\varepsilon)}=\varepsilon \frac{m(2-\varepsilon)+\varepsilon-1}{m-\varepsilon(1-\varepsilon)},
$$

and then:

$$
\lim _{m \rightarrow+\infty}\left(2^{m}(1-m s)\right)=2
$$

hence via (5.53) and (5.54) we get:

$$
\int_{\Omega} \frac{|B|^{3}}{2+\lambda} d x \leq \delta a^{2}(t)+C(\delta)\left(\left(1+Z^{2}(t)\right)^{2}+m\left(\frac{m}{\varepsilon}\right)^{\frac{1}{\beta-1}}\left(1+Z^{2}(t)\right)^{2+\frac{1-m s}{m(s+1)-2}}\right) .
$$

Now, consider the last term in (5.50):

$$
I_{2}=\int_{\Omega}|B|\left(U_{x}^{2}+U_{y}^{2}+V_{x}+V_{y}^{2}\right) \leq\|B\|_{L^{2 m}(\Omega)}\left(\int_{\Omega}\left(|\nabla U|^{2}+|\nabla V|^{2}\right)^{\frac{2 m}{2 m-1}} d x\right)^{1-\frac{1}{2 m}}
$$

Recalling the relation $3>\frac{4 m}{2 m-1}>2, m>2$, from the properties of elliptic system [24] we derive the inequality:

$$
\left(\int_{\Omega}\left(|\nabla U|^{2}+|\nabla V|^{2}\right)^{\frac{2 m}{2 m-1}} d x\right)^{1-\frac{1}{2 m}} \leq C\left(\|\operatorname{div} u\|_{L^{\frac{4 m}{2 m-1}}(\Omega)}^{2}+\|A\|_{L^{\frac{4 m}{2 m-1}}(\Omega)}^{2}\right) .
$$

Thus the previous inequality furnish the estimate:

$$
I_{2} \leq C\|B\|_{L^{2 m}(\Omega)}\left(\|\operatorname{div} u\|_{L^{\frac{4 m}{2 m-1}}(\Omega)}^{2}+\|A\|_{L^{\frac{4 m}{2 m-1}}(\Omega)}^{2}\right) .
$$


Next we have as $A$ vanishes on the boundary of the domain $\Omega$, we applythe GagliardoNiremberg inequality:

$$
\begin{aligned}
& \|A\|_{L^{\frac{4 m}{2 m-1}}(\Omega)}^{2} \leq C\|A\|_{L^{2}(\Omega)}^{2-\frac{1-\varepsilon}{m(1-2 \varepsilon)}}\|\nabla A\|_{L^{2(1-\varepsilon)}(\Omega)}^{\frac{1-\varepsilon}{m(1-2 \varepsilon)}}, \\
& \leq C Z^{2-\frac{1-\varepsilon}{m(1-2 \varepsilon)}}(t)\left(\left(\frac{1}{\varepsilon}\right)^{\frac{1}{2(\beta-1)}} a(t)\right)^{\frac{1-\varepsilon}{m(1-2 \varepsilon)}} \leq C Z^{2-\frac{1-\varepsilon}{m(1-2 \varepsilon)}}(t)(a(t))^{\frac{1-\varepsilon}{m(1-2 \varepsilon)}} .
\end{aligned}
$$

Since $B=(2+\lambda) \operatorname{div} u-P$, estimate (5.38) provides:

$$
\begin{aligned}
\|\operatorname{div} u\|_{L^{\frac{4 m}{2 m-1}}(\Omega)}^{2} & =\left\|\frac{B}{2+\lambda}+\frac{P}{2+\lambda}\right\|_{L^{\frac{4 m}{2 m-1}}(\Omega)}^{2}, \\
& \leq C\left(\left\|\frac{B}{2+\lambda}\right\|_{L^{\frac{4 m}{2 m-1}}(\Omega)}^{2}+1\right) .
\end{aligned}
$$

We can now deal with the right-hand side of (5.58) as follows:

$$
\begin{aligned}
& \left\|\frac{B}{2+\lambda}+\frac{P}{2+\lambda}\right\|_{L^{\frac{4 m}{2 m-1}(\Omega)}}^{2} \leq\left(\int_{\Omega} \frac{|B|^{\frac{2 m(2 m-3)}{(m-1)(2 m-1)}}}{2+\lambda}|B|^{\frac{2 m}{(m-1)(2 m-1)}} d x\right)^{1-\frac{1}{2 m}} \\
& \leq\|B\|_{L^{2 m}(\Omega)}^{\frac{1}{m-1}}\left(\int_{\Omega} \frac{|B|^{2}}{(2+\lambda)^{\frac{(m-1)(2 m-1)}{m(2 m-3)}}} d x\right)^{\frac{2 m-3}{2 m-2}} \leq\|B\|_{L^{2 m}(\Omega)}^{\frac{1}{m-1}}\left(\int_{\Omega} \frac{|B|^{2}}{(2+\lambda)} d x\right)^{\frac{2 m-3}{2 m-2}} \\
& \leq\|B\|_{L^{2 m}(\Omega)}^{\frac{1}{m-1}}(Z(t))^{\frac{2 m-3}{2 m-2}}=\|B\|_{L^{2 m}(\Omega)}^{\frac{1}{m-1}}(Z(t))^{2-\frac{1}{m-1}}
\end{aligned}
$$

Thus,

$$
\|\operatorname{div} u\|_{L^{\frac{4 m}{2 m-1}}(\Omega)}^{2} \leq C\left(1+(Z(t))^{2-\frac{1}{m-1}}\|B\|_{L^{2 m}(\Omega)}^{\frac{1}{m-1}}\right) .
$$

Using estimates (5.57) and (5.59), from (5.56) we have:

$$
I_{2} \leq C\|B\|_{L^{2 m}(\Omega)}\left(1+Z^{2-\frac{1-\varepsilon}{m(1-2 \varepsilon)}}(t)(a(t))^{\frac{1-\varepsilon}{m(1-2 \varepsilon)}}+Z^{2-\frac{1}{m-1}}(t)\|B\|_{L^{2 m}(\Omega)}^{\frac{1}{m-1}}\right) .
$$

Using estimate (5.52) for $\|B\|_{L^{2 m}(\Omega)}$, we finally get:

$$
\begin{aligned}
& I_{2} \leq C\left((Z(t))^{3-\frac{1-\varepsilon}{m(1-2 \varepsilon)}}(a(t))^{\frac{1-\varepsilon}{m(1-2 \varepsilon)}}+Z(t)+Z^{3}(t)\right. \\
& +m^{\frac{1}{2}}\left(\frac{m}{\varepsilon}\right)^{\frac{1-s}{2(\beta-1)}} Z^{2+s-\frac{1-\varepsilon}{m(1-2 \varepsilon)}}(t)(a(t))^{1-s+\frac{1-\varepsilon}{m(1-2 \varepsilon)}} \\
& \left.+m^{\frac{1}{2}}\left(\frac{m}{\varepsilon}\right)^{\frac{m(1-s)}{2(\beta-1)(m-1)}} Z^{2+\frac{m s-1}{m-1}}(t)(a(t))^{\frac{m(1-s)}{m-1}}+m^{\frac{1}{2}}\left(\frac{m}{\varepsilon}\right)^{\frac{1-s}{2(\beta-1)}} Z^{s}(t)(a(t))^{1-s}\right) .
\end{aligned}
$$


Using Young's inequality, we treat the summand in (5.60) as follows:

$$
\begin{aligned}
& C(Z(t))^{3-\frac{1-\varepsilon}{m(1-2 \varepsilon}}(a(t))^{\frac{1-\varepsilon}{m(1-2 \varepsilon)}} \leq \delta a^{2}(t)+C\left(1+Z^{2}(t)\right)^{2}, \\
& C\left(Z(t)+Z^{3}(t)\right) \leq C\left(1+Z^{2}(t)\right)^{2} \\
& C m^{\frac{1}{2}}\left(\frac{m}{\varepsilon}\right)^{\frac{1-s}{2(\beta-1)}} Z^{2+s-\frac{1-\varepsilon}{m(1-2 \varepsilon)}}(t)(a(t))^{1-s+\frac{1-\varepsilon}{m(1-2 \varepsilon)}} \\
& \quad \leq \delta a^{2}(t)+C m\left(\frac{m}{\varepsilon}\right)^{\frac{1}{\beta-1}}\left(1+Z^{2}(t)\right)^{2+\frac{1-m s+(2 m s-1) \varepsilon}{(1+s) m(1-2 \varepsilon)-1+\varepsilon}}, \\
& C m^{\frac{1}{2}}\left(\frac{m}{\varepsilon}\right)^{\frac{1-s}{2(\beta-1)}} Z^{s}(t)(a(t))^{1-s} \leq \delta a^{2}(t)+C m\left(\frac{m}{\varepsilon}\right)^{\frac{1}{\beta-1}}\left(1+Z^{2}(t)\right), \\
& m^{\frac{1}{2}}\left(\frac{m}{\varepsilon}\right)^{\frac{m(1-s)}{2(\beta-1)(m-1)}} Z^{2+\frac{m s-1}{m-1}}(t)(a(t))^{\frac{m(1-s)}{m-1}} \\
& \quad \leq \delta a^{2}(t)+C m\left(\frac{m}{\varepsilon}\right)^{\frac{1}{\beta-1}}\left(1+Z^{2}(t)\right)^{2+\frac{1-m s}{(1+s) m-2}} .
\end{aligned}
$$

Here $\delta$ is a small positive constant to be mentioned below. From inequality (5.60) we derive that:

$$
\begin{aligned}
& I_{2} \leq \delta a^{2}(t)+C\left(m\left(\frac{m}{\varepsilon}\right)^{\frac{1}{\beta-1}}\left(1+Z^{2}(t)\right)^{2+\frac{1-m s+(2 m s-1) \varepsilon}{(1+s) m(1-2 \varepsilon)-1+\varepsilon}}\right. \\
& +\left(1+Z^{2}(t)\right)^{2}+m\left(\frac{m}{\varepsilon}\right)^{\frac{1}{\beta-1}}\left(1+Z^{2}(t)\right)+m\left(\frac{m}{\varepsilon}\right)^{\frac{1}{\beta-1}}\left(1+Z^{2}(t)\right)^{2+\frac{1-m s}{(1+s) m-2}}
\end{aligned}
$$

From (5.55) and (5.61), and inequality (5.50) we have:

$$
\begin{aligned}
& \frac{1}{2} \frac{d}{d t}\left(Z^{2}(t)+a^{2}(t)\right) \leq \delta a^{2}(t)+C(\delta)\left(1+Z^{2}(t)\right)^{2+\frac{\varepsilon}{3-\varepsilon}}\left(\frac{1}{\varepsilon}\right)^{\frac{1}{\beta-1}} \\
& +C\left(1+\delta a^{2}(t)\right)+C C(\delta)\left(1+Z^{2}(t)\right)^{2}+C C(\delta)\left(m\left(\frac{m}{\varepsilon}\right)^{\frac{1}{\beta-1}}\left(1+Z^{2}(t)\right)^{2+\frac{1-m s}{m(s+1)-2}}\right. \\
& +4 \delta a^{2}(t)+C\left(\left(\left(1+Z^{2}(t)\right)^{2}+m\left(\frac{m}{\varepsilon}\right)^{\frac{1}{\beta-1}}\left(1+Z^{2}(t)\right)^{2+\frac{1-m s+(2 m s-1) \varepsilon}{(1+s) m(1-2 \varepsilon)-1+\varepsilon}}\right.\right. \\
& \left.+m\left(\frac{m}{\varepsilon}\right)^{\frac{1}{\beta-1}}\left(1+Z^{2}(t)\right)^{2}+m\left(\frac{m}{\varepsilon}\right)^{\frac{1}{\beta-1}}\left(1+Z^{2}(t)\right)^{2+\frac{1-m s}{m(s+1)-2}}\right) .
\end{aligned}
$$

Choose $\delta>0$ such that:

$$
5 \delta+\delta C=\frac{1}{2}
$$

Since $s=\frac{(1-\varepsilon)^{2}}{m-\varepsilon(1-\varepsilon)}$ and $\varepsilon=2^{-m}, m>2$, we have:

$$
\frac{1-m s}{m(s+1)-2} \leq 4 \varepsilon, \frac{1-m s+(2 m s-1) \varepsilon}{(1+s) m(1-2 \varepsilon)-1+\varepsilon} \leq 4 \varepsilon \text { and } \frac{\varepsilon}{1-3 \varepsilon} \leq 4 \varepsilon .
$$

Then by (5.62) and the fact that $Z^{2}(t) \in L^{1}(0, T)$, we obtain the inequality with $0<\bar{T}<\frac{T}{2}$ :

$$
\frac{1}{2} \frac{d}{d t}\left(1+Z^{2}(t)\right)+a^{2}(t) \leq m\left(\frac{m}{\varepsilon}\right)^{\frac{1}{\beta-1}}\left(1+Z^{2}(t)\right)^{2+4 \varepsilon} .
$$

From (5.63) we have for $0 \leq t<T$ :

$$
\frac{1}{\left(1+Z^{2}(t)\right)^{4 \varepsilon}}-\frac{1}{\left(1+Z^{2}(\bar{T})\right)^{4 \varepsilon}}+C m \varepsilon\left(\frac{m}{\varepsilon}\right)^{\frac{1}{\beta-1}} \geq 0 .
$$


REMARK 8. Let us point out that the last inequality is better than in [34] and allows us to assume only $\beta>2$.

Now, take $N>2$ such that:

$$
1-C N \varepsilon\left(\frac{N}{\varepsilon}\right)^{\frac{1}{\beta-1}}\left(1+Z^{2}(\overline{0})\right)^{4 \varepsilon} \geq \frac{1}{2}, \varepsilon=2^{-N} .
$$

Here the fact that $\beta>2$ allows to conclude and by this fact improve the results of [34]. We get finally that for $0 \leq t<T$ :

$$
Z^{2}(t) \leq 2^{2^{N-2}}\left(1+Z^{2}(0)\right)-1, \quad t \in[0, T] .
$$

Now, from inequality (5.63) we get moreover that:

$$
\int_{0}^{T} a^{2}(t) d t \leq C
$$

Now by estimate (5.38) for the density, there exists a positive constant $C$ depending continuously on the data of the problem and such that:

$$
\begin{aligned}
& \sup _{0<t<T} \int_{\Omega}\left((\operatorname{curl} u)^{2}+\frac{1}{2+\lambda(\rho)}((2+\lambda(\rho)) \operatorname{div} u-P(\rho))^{2}\right)(t, x) d x \leq C, \\
& \sup _{0<t<T} \int_{\Omega}\left((\operatorname{curl} u)^{2}+(2+\lambda(\rho))(\operatorname{div} u)^{2}\right)(t, x) d x \leq C, \\
& \int_{0}^{T} \int_{\Omega} \frac{\left(A_{y}+B_{x}\right)^{2}+\left(-A_{x}+B_{y}\right)^{2}}{\rho} d x d y \leq C .
\end{aligned}
$$

The rest of the proof follows exactly the same lines than in [34] and then we refer to [34].

6. Proof of Theorem 1.2. We are interested in proving the existence of global strong solution with small initial data for the system (1.5). $W$ is the semi group associated to the following linear system $(6.67)$ with $\left(q_{1}, m_{1}\right)$ a solution:

$$
\left\{\begin{array}{l}
\partial_{t} q_{1}+\operatorname{div} m_{1}-\mu \Delta q_{1}=0 \\
\partial_{t} m_{1}-\mu \Delta m_{1}+a m_{1}=0
\end{array}\right.
$$

Denote $q=q_{L}+\bar{q}, m=m_{L}+\bar{m}$ where $\left(q_{L}, m_{L}\right)$ are solutions of (6.67) with initial data $\left(q_{0}, m_{0}\right)$. We are going to use a contracting mapping argument for the function $\psi$ defined as follows:

$$
\psi(\bar{q}, \bar{m})=\int_{0}^{t} W(t-s)\left(\begin{array}{c}
0 \\
G\left(q_{L}+\bar{q}, m_{L}+\bar{m}\right)
\end{array}\right) d s .
$$

where The non linear terms $G$ is defined as follows:

$$
G(q, m)=-\operatorname{div}\left(\frac{m}{1+q} \otimes q\right)
$$

We are going to check that we can apply a fixed point theorem for the function $\psi$ in $E^{\frac{N}{2}}$ defined below, the proof is divided in two step the stability of $\psi$ for a ball $B(0, R)$ in $E^{\frac{N}{2}}$ and the contraction property. We define $E^{\frac{N}{2}}$ by:

$$
E^{\frac{N}{2}}=\left(\widetilde{C}\left(B_{2,1}^{\frac{N}{2}}\right) \cap \widetilde{L}^{1}\left(B_{2, \infty}^{\frac{N}{2}+2}\right)\right) \times\left(\widetilde{C}\left(B_{2,1}^{\frac{N}{2}-1}\right) \cap \widetilde{L}^{1}\left(B_{2,1}^{\frac{N}{2}-1} \cap B_{2,1}^{\frac{N}{2}+1}\right)\right)^{N} .
$$


1) First step, stability of $B(0, R)$ :. Let:

$$
\eta=\left\|q_{0}\right\|_{B_{2,1}^{\frac{N}{2}}}+\left\|m_{0}\right\|_{B_{2,1}^{\frac{N}{2}-1}} .
$$

We are going to show that $\psi$ maps the ball $B(0, R)$ into itself if $R$ is small enough. According to proposition 3.3, we have:

$$
\left\|W(t, \cdot) *\left(\begin{array}{c}
q_{0} \\
m_{0}
\end{array}\right)\right\|_{E^{\frac{N}{2}}} \leq C\left(\left\|q_{0}\right\|_{B_{2,1}^{\frac{N}{2}}}+\left\|m_{0}\right\|_{B_{2,1}^{\frac{N}{2}}}\right)=C \eta .
$$

According to the proposition 3.3 it implies also that it exists $C>0$ such that:

$$
\|\psi(\bar{q}, \bar{m})\|_{E^{\frac{N}{2}}} \leq C\|G(q, m)\|_{\widetilde{L}^{1}\left(B_{2,1}^{\frac{N}{2}-1}\right)} .
$$

Making the assumption:

$$
\|q\|_{L^{\infty}\left(\mathbb{R}^{+} \times \mathbb{R}^{N}\right)} \leq \frac{1}{2}
$$

The main task consists in using the propositions 3.2 to obtain estimates on $\|G(q, u)\|_{\widetilde{L}^{1}\left(B_{2,1}^{\frac{N}{2}-1}\right)}$. Hence by proposition 3.2 and lemma 4 it yields:

$$
\begin{aligned}
\left\|\operatorname{div}\left(\frac{m}{1+q} \otimes m\right)\right\|_{L^{1}\left(B_{2,1}^{\frac{N}{2}}-1\right)} & \leq\left\|\frac{m}{1+q} \otimes m\right\|_{L^{1}\left(B_{2,1}^{\frac{N}{2}-1}\right)}, \\
& \leq C\|m\|_{L_{T}^{2}\left(B_{2,1}^{2}\right)}^{2 \frac{N}{2}}\left(\left\|\frac{1}{1+q}-1\right\|_{L_{T}^{\infty}\left(B_{2,1}^{\frac{N}{2}}\right)}+1\right), \\
& \leq C\|m\|_{L_{T}^{2}\left(B_{2,1}^{\frac{N}{2}}\right)}^{2}\left(\|q\|_{L_{T}^{\infty}\left(B_{2,1}^{\frac{N}{2}}\right)}+1\right) .
\end{aligned}
$$

We are now going to assume that $(q, u)$ belongs in the ball $B(0, R)$ of $E^{\frac{N}{2}}$ with $R>0$. Combining the estimates $(6.70),(6.71),(6.72)$ and $(6.73)$ we get:

$$
\|\psi(\bar{q}, \bar{m})\|_{E^{\frac{N}{2}}} \leq C((C+1) \eta+R)^{2} .
$$

By choosing $R$ and $\eta$ small enough we have:

$$
C((C+1) \eta+R)^{2} \leq R
$$

To do this it suffices in a first time to verify the assumption (6.72), let $c$ be a constant such that $\|\cdot\|_{B_{2,1}^{\frac{N}{2}}} \leq c$ implies $\|\cdot\|_{L^{\infty}} \leq \frac{1}{2}$, we choose $R \leq c$ and finally we can choose $R$ and $\eta$ such that:

$$
R \leq \inf \left((3 C)^{-1}, c, 1\right), \text { and } \eta \leq \frac{\inf (R, c)}{C+1} .
$$

It implies that the ball $B(0, R)$ of $E^{\frac{N}{2}}$ is stable under $\psi$, indeed we have:

$$
\psi(B(0, R)) \subset B(0, R) .
$$


2) Second step: Property of contraction. We consider $\left(\bar{q}_{1}, \bar{m}_{1}\right),\left(\bar{q}_{2}, \bar{m}_{2}\right)$ in $B(0, R)$ and we are interested in verifying that $\psi$ is a contraction. According to the proposition 3.3 we have:

$$
\left\|\psi\left(\bar{q}_{2}, \bar{m}_{2}\right)-\psi\left(\bar{q}_{1}, \bar{m}_{1}\right)\right\|_{E^{\frac{N}{2}}} \leq C\left\|G\left(q_{2}, m_{2}\right)-G\left(q_{1}, m_{1}\right)\right\|_{\widetilde{L}^{1}\left(B_{2,1}^{\frac{N}{2}-1}\right)} .
$$

We set:

$$
\delta q=\bar{q}_{2}-\bar{q}_{1} \quad \text { and } \quad \delta m=\bar{m}_{2}-\bar{m}_{1} .
$$

We have then:

$$
G\left(q_{2}, m_{2}\right)-G\left(q_{1}, m_{1}\right)=\operatorname{div}\left(m_{1} \otimes m_{1}\left(\frac{q_{2}}{1+q_{2}}-\frac{q_{1}}{1+q_{1}}\right)-\frac{m_{2} \otimes \delta m+\delta m \otimes m_{1}}{1+q_{2}}\right) .
$$

Applying proposition 3.2 and lemma 4 we obtain:

$$
\begin{aligned}
& \left\|G\left(q_{2}, m_{2}\right)-G\left(q_{1}, m_{1}\right)\right\|_{\widetilde{L}^{1}\left(B_{2,1}^{\frac{N}{2}-1}\right)} \\
& \leq C\|(\delta q, \delta m)\|_{E^{\frac{N}{2}}}\left(\left\|\left(\bar{q}_{1}, \bar{m}_{1}\right)\right\|_{E^{\frac{N}{2}}}+2\left\|\left(q_{L}, m_{L}\right)\right\|_{E^{\frac{N}{2}}}\right) .
\end{aligned}
$$

Now, if $(R, \eta)$ satisfies $(6.76)$ (for a greater constant $C$ if necessary), it yields:

$$
\left\|\psi\left(\bar{q}_{2}, \bar{m}_{2}\right)-\psi\left(\bar{q}_{1}, \bar{m}_{1}\right)\right\|_{E^{\frac{N}{2}}} \leq \frac{1}{2}\|(\delta q, \delta m)\|_{E^{\frac{N}{2}}}
$$

Since $E^{\frac{N}{2}}$ is a Banach space, we have proved via the fixed point theorem the existence of a unique global solution of the system $(1.5)$ in the ball $B(0, R)$ of $E^{\frac{N}{2}}$. It concludes the proof.

\section{REFERENCES}

[1] V. I. ARnold And B. A. Khesin, Topological methods in hydrodynamics, volume 125 od Applied Mathematical Sciences - Springer Verlag, New-York, 1998.

[2] D-.M. Anderson, G-.B Mcfadden and A-.A. Wheller, Diffuse-interface methods in fluid mech, in Annal review of fluid mechanics, Vol. 30, pages 139-165. Annual Reviews, Palo Alto, CA, 1998

[3] H. Bahouri, J.-Y. Chemin, And R. Danchin, Fourier analysis and nonlinear partial differential equations, Grundlehren der mathematischen Wissenschaften, 343, Springer Verlag, 2011.

[4] J.-M. BonY, Calcul symbolique et propagation des singularités pour les équations aux dérivées partielles non linéaires, Annales Scientifiques de l'école Normale Supérieure, 14 (1981), pp. 209-246.

[5] D. Bresch, B. Desjardins And C-. K. Lin, On some compressible fluid models: Korteweg,lubrication and shallow water systems, Comm. Partial Differential Equations, 28(34) (2003), pp. 843-868.

[6] J-.W. Cahn and J-.E. Hilliard, Free energy of a nonuniform system, I. Interfacial free energy, J. Chem. Phys., 28 (1998), pp. 258-267.

[7] R. Coifman, P.-L. Lions, Y. Meyer and S. Semmes, Compensated-compactness and Hardy spaces, J. Math. Pures Appl., 72 (1993), pp. 247-286.

[8] R. Danchin and B. Desjardins, Existence of solutions for compressible fluid models of Korteweg type, Annales de l'IHP, Analyse non linéaire, 18 (2001), pp. 97-133.

[9] J-. E. Dunn And J. SeRrin, On the thermomechanics of interstitial working, Arch. Rational Mech. Anal., 88:2 (1985), pp. 95-133.

[10] E. Gagliardo, Ulteriori proprieta di alcune classi di funzioni in piu variablil, Ricerche Mat., 8 (1959), pp. 24-51.

[11] K. K. Golovkin, On embedding theorems, Dokl. Akad. Nausk SSSR, 134 (1960), pp. 19-22. 
[12] M.-E. Gurtin, D. Poligone And J. Vinals, Two-phases binary fluids and immiscible fluids described by an order parameter, Math. Models Methods Appl. Sci., 6:6 (1996), pp. 815831.

[13] B. Haspot, Global existence of strong solution for shallow water system with large initial data on the irrotational part, preprint arXiv:1201.5456 (2012).

[14] B. Haspot, Global existence of strong solution for the Saint-Venant system with large initial data on the irrotational part, C. R. Math. Acad. Sci. Paris, 350 (2012), pp. 229-332.

[15] B. HASPOT, Existence of global strong solutions for the barotropic Navier Stokes system system with large initial data on the rotational part of the velocity, C. R. Math. Acad. Sci. Paris., 350 (2012), pp. 487-492.

[16] B. HASPOT, Existence of global strong solution for Korteweg system with large infinite energy initial data, preprint (arxiv 2013).

[17] B. HAspot, New entropy for Korteweg's system, existence of global weak solution and ProdiSerrin theorem, preprint (arxiv March 2011).

[18] B. HAspot, Existence of solutions for compressible fluid models of Korteweg type, Annales Mathématiques Blaise Pascal, 16 (2009), pp. 431-481.

[19] B. HASPOT, Existence of weak solution for compressible fluid models of Korteweg type, Journal of Mathematical Fluid Mechanics, 13:2 (2011), pp. 223-249 .

[20] B. НASPOT, Cauchy problem for viscous shallow water equations with a term of capillarity, accepted in Hyperbolic Problems: Theory, Numerics and Application PSAPM volume, edited by Drs. Tadmor, Liu, Tzavaras.

[21] X. Huang And J. LI, Existence and Blowup Behavior of Global Strong Solutions to the TwoDimensional Barotropic Compressible Navier-Stokes System with Vacuum and Large Initial Data, preprint arxiv 2012.

[22] A. Jüngel, Global weak solutions to compressible Navier-Stokes equations for quantum fluids, SIAM Journal on Mathematical Analysis, 42:3 (2011), pp. 1025-1045.

[23] V. P. ILIN, Some inequalities in function spaces and their application to studying convergence of variational methods, Trudy Mat. Inst. Akad. Nauk SSSR, 53 (1959), pp. 64-127.

[24] O. A. Ladhyzhenskaya and N. N. URAltseva, Linear and quasilinear equations of parabolic type (in russian), Nauka, Moscow (1967).

[25] O. A. Ladhyzhenskaya And N. N. URALTSEVA, Linear and quasilinear equations of parabolic type (in russian), Nauka, Moscow (1973).

[26] P.-L. Lions, Mathematical Topics in Fluid Mechanics, Vol 2, Compressible models, Oxford University Press, (1996).

[27] J. Pedlosky, Geophysical Fluid Dynamics, Berlin Heidelberg-New York, Springer Verlag, 1987.

[28] S. I. Pokhozhaev, On SL Sobolev's embedding theorem in the case $p l=n$, Dokl. NauchnoTekhn. Konf. MEI, (1965), pp. 158-170.

[29] T. Runst And W. Sickel, Sobolev spaces of fractional order, Nemytskij operators, and nonlinear partial differential equations, volume 3 of De Gruyter series in nonlinear analysis and applications, Berlin 1996.

[30] E. M. STEIN, Singular Integrals and differentiability properties of functions, Princeton Univ. Press, 1970.

[31] J. Simon, Compact sets in the space $L^{p}(0, T ; B)$, Ann. Mat. Pura Appl., 146 (1987), pp. 65-96.

[32] G. Thlenti, Best constant in Sobolev inequality, Ann. Mat. Pura. Appl., (4), 110 (1976), pp. 353-372.

[33] C. Truedelland And W. Noll, The nonlinear field theories of mechanics, Springer-Verlag, Berlin, second edition, 1992.

[34] V. A. VAigant AND A. V Kazhikhov, On existence of global solutions to the two-dimensional Navier-Stokes equations for a compressible viscous fluid, Siberian Mathematical Journal, 36:6 (1995).

[35] V. I. YUdovich, On some estimates connected with integral operators and solutions of elliptic equations, Dokl. Akad. Nauk SSSR, 138:4 (1961), pp. 805-808.

[36] V. A. WEIGANT, Example of non-existence in the large for the problem of the existence of solutions of Navier-Stokes equations for compressible viscous barotropic fluids, Dokl. Akad. Nauk, 339 (1994), pp. 155-156 (in Russian). 